\title{
THE PRINCIPAL EIGENVALUE OF THE $\infty$-LAPLACIAN WITH THE NEUMANN BOUNDARY CONDITION
}

\author{
Stefania PATRIZI ${ }^{1}$
}

\begin{abstract}
We prove the existence of a principal eigenvalue associated to the $\infty$-Laplacian plus lower order terms and the Neumann boundary condition in a bounded smooth domain. As an application we get uniqueness and existence results for the Neumann problem and a decay estimate for viscosity solutions of the Neumann evolution problem.
\end{abstract}

Mathematics Subject Classification. 35J25, 35D40, 35P30, 35J60.

Received July 14, 2008.

Published online May 10, 2010.

\section{INTRODUCTION}

In this paper we study the maximum principle, the principal eigenvalue, regularity, existence and uniqueness for viscosity solutions of the Neumann boundary value problem

$$
\begin{cases}\Delta_{\infty} u+b(x) \cdot D u+(c(x)+\lambda) u=g(x) & \text { in } \Omega \\ \frac{\partial u}{\partial \bar{n}}=0 & \text { on } \partial \Omega,\end{cases}
$$

where $\Omega$ is a bounded smooth domain, $\vec{n}(x)$ is the exterior normal to the domain $\Omega$ at $x, b, c$ and $g$ are continuous functions on $\bar{\Omega}, \lambda \in \mathbb{R}$ and

$$
\Delta_{\infty} u=\left\langle D^{2} u \frac{D u}{|D u|}, \frac{D u}{|D u|}\right\rangle,
$$

for $u \in C^{2}(\Omega)$, is the 1-homogeneous version of the $\infty$-Laplacian.

The $\infty$-Laplacian, which arises from the optimal Lipschitz extension problem, see [2], appears also in the Monge-Kantorovich mass transfer problem, see [7], and recently, some authors have introduced a game theoretic interpretation of it, see [18].

We define and investigate the properties of the principal eigenvalue of the operator

$$
-\left(\Delta_{\infty}+b(x) \cdot D+c(x)\right),
$$

with the Neumann boundary condition and as an application, we get existence and uniqueness results for (1.1) and a decay estimate for the solution of the associated evolution problem.

\footnotetext{
Keywords and phrases. $\infty$-Laplacian, Neumann boundary condition, principal eigenvalue, viscosity solutions.

1 SAPIENZA Università di Roma, Dipartimento di Matematica, Piazzale A. Moro 2, 00185 Roma, Italy.

patrizi@mat .uniroma1.it
} 
In their famous work [3], Berestycki et al. defined the principal eigenvalue $\lambda_{1}$ of a general linear uniformly elliptic operator $-L$ where

$$
L[u]=\operatorname{tr}\left(A(x) D^{2} u\right)+b(x) \cdot D u+c(x) u,
$$

in a bounded domain $\Omega$, as the supremum of those $\lambda$ for which there exists a positive supersolution of $L[u]+$ $\lambda u=0$. In that paper, they showed that $\lambda_{1}$ is the first eigenvalue of $L$, i.e., for any eigenvalue $\lambda \neq \lambda_{1}$, $\operatorname{Re}(\lambda)>\lambda_{1}$; moreover $\lambda_{1}$ can be characterized as the supremum of those $\lambda$ for which the operator $L+\lambda I$ satisfies the maximum principle, i.e., for any $\lambda<\lambda_{1}$, if $u$ is a subsolution of $L[u]+\lambda u=0$ and $u \leq 0$ on $\partial \Omega$ then $u \leq 0$ in $\Omega$. They established other properties of the first eigenvalue, such as simplicity and stability.

In view of its relation with the maximum and the comparison principles, the concept of principal eigenvalue has been extended to nonlinear operators to study the associated boundary value problems. That has been done for the variational operators, such that the $p$-Laplacian, through the method of minimization of the so called nonlinear Rayleigh quotient, see e.g. $[1,14]$. That method uses heavily the variational structure and cannot be applied to operators which have not this property. An important step in the study of the eigenvalue problem for general nonlinear operators was made by Lions in [15]. In that paper, using probabilistic and analytical methods, he showed the existence of principal eigenvalues for the uniformly elliptic Hamilton-Jacobi-Bellman operator. Very recently, many authors, inspired by [3], have developed an eigenvalue theory for fully nonlinear operators which are non-variational. The Pucci's extremal operators have been treated by Quaas [19] and Busca et al. [5]. Their results have been extended to more general fully nonlinear convex uniformly elliptic operators in [20] by Quaas and Sirakov. See also the work of Ishii and Yoshimura [10] for non-convex operators.

Issues similar to those of this paper have been studied by Birindelli and Demengel in [4] and the author of this note in [16] where respectively the Dirichlet and the Neumann eigenvalue problem is treated for degenerate or singular elliptic operators $F\left(x, D u, D^{2} u\right)$ plus lower order terms. In these papers, among other assumptions, $F$ is required to satisfied

$$
a|p|^{\alpha} \operatorname{tr} N \leq F(x, p, M+N)-F(x, p, M) \leq A|p|^{\alpha} \operatorname{tr} N,
$$

with $\alpha>-1$, for $x \in \bar{\Omega}, p \in \mathbb{R}^{N} \backslash\{0\}$, and $M, N$ symmetric matrices with $N \geq 0$. Typical examples are given by $|D u|^{\alpha} \mathcal{M}_{a, A}\left(D^{2} u\right), \alpha>-1$, where $\mathcal{M}_{a, A}\left(D^{2} u\right)$ is one of the Pucci's operator, the $p$-Laplacian and some non-variational generalizations of it. Because of its strong degeneracy, the $\infty$-Laplacian does not satisfy (1.3), so it is not covered by [4] or [16].

The existence of a principal eigenvalue defined as in [3] for the $\infty$-Laplacian with the Dirichlet boundary condition has been treated by Juutinen in [11] together with many other questions. We want to mention that there exists also a different approach to investigate the eigenvalue problem for (1.2) which consists in studying the asymptotic behavior, as $p \rightarrow \infty$, of the $p$-Laplacian eigenvalue equation, see $[8,13]$. This second method uses the variational formulation of the approximate problems and leads to a different limit eigenvalue problem, see $[11]$.

Following the ideas of [3], we define the principal eigenvalue as

$$
\begin{aligned}
\bar{\lambda}:=\sup \{\lambda \in \mathbb{R} \mid \exists v>0 \text { on } \bar{\Omega} \text { bounded viscosity supersolution of } \\
\left.\Delta_{\infty} v+b(x) \cdot D v+(c(x)+\lambda) v=0 \text { in } \Omega, \frac{\partial v}{\partial \vec{n}}=0 \text { on } \partial \Omega\right\} .
\end{aligned}
$$

Here we adopt the notion of viscosity solution given in [4], in which it suffices to test with functions which have gradient different from 0 .

The quantity $\bar{\lambda}$ is well defined since the above set is not empty; indeed, $-|c|_{\infty}$ belongs to it, being $v(x) \equiv 1$ a corresponding supersolution. Furthermore it is an interval because if $\lambda$ belongs to it then so does any $\lambda^{\prime}<\lambda$.

One of the scope of this work is to prove that $\bar{\lambda}$ is an "eigenvalue" for $-\left(\Delta_{\infty}+b(x) \cdot D+c(x)\right)$ which admits a positive "eigenfunction". As in the linear case it can be characterized as the supremum of those $\lambda$ 
for which $\Delta_{\infty}+b(x) \cdot D+c(x)+\lambda$ with the Neumann boundary condition satisfies the maximum principle. As a consequence, $\bar{\lambda}$ is the least "eigenvalue", i.e., the least number for which there exists a non-zero solution of

$$
\begin{cases}\Delta_{\infty} u+b(x) \cdot D u+(c(x)+\lambda) u=0 & \text { in } \Omega \\ \frac{\partial u}{\partial \vec{n}}=0 & \text { on } \partial \Omega .\end{cases}
$$

These results are applied to obtain existence and uniqueness for the boundary value problem (1.1).

Remark that since $\Delta_{\infty}(-u)=-\Delta_{\infty} u, \bar{\lambda}$ can be defined also in the following way

$$
\begin{aligned}
\bar{\lambda}=\sup \{\lambda \in \mathbb{R} \mid \exists u<0 \text { on } \bar{\Omega} \text { bounded viscosity subsolution of } \\
\\
\left.\Delta_{\infty} u+b(x) \cdot D u+(c(x)+\lambda) u=0 \text { in } \Omega, \frac{\partial u}{\partial \vec{n}}=0 \text { on } \partial \Omega\right\} .
\end{aligned}
$$

For a fully nonlinear operator, $\bar{\lambda}$ defined as in (1.4) may be different from the quantity defined as in (1.5), see [17].

The paper is organized as follows. In the next section we give assumptions and precise the concept of solution adopted. In Section 3 we establish a Lipschitz regularity result for viscosity solutions of (1.1). Section 4 is devoted to the maximum principle for subsolutions of (1.1). In Section 4.1 we show that it holds (even for more general boundary conditions) for $\Delta_{\infty}+b(x) \cdot D+c(x)$ if $c(x) \leq 0$ and $c \not \equiv 0$, see Theorem 4.4. One of the main result of the paper is that the maximum principle holds for $\Delta_{\infty}+b(x) \cdot D+c(x)+\lambda$ for any $\lambda<\bar{\lambda}$, as we show in Theorem 4.8 of Section 4.2. In particular it holds for $\Delta_{\infty}+b(x) \cdot D+c(x)$ if $\bar{\lambda}>0$. Following the example given in [16] we show that the result of Theorem 4.8 is stronger than that of Theorem 4.4, i.e., that there exist some functions $c(x)$ changing sign in $\Omega$ for which the principal eigenvalue of $\Delta_{\infty}+b(x) \cdot D+c(x)$ is positive and then for which the maximum principle holds.

In Section 5 we show some existence and comparison theorems. In particular, we prove that the Neumann problem (1.1) is solvable for any right-hand side if $\lambda<\bar{\lambda}$.

Finally, in Section 6 we prove a decay estimate for solutions of the Neumann evolution problem.

\section{Assumptions And Definitions}

We denote by $S(N)$ the space of symmetric matrices on $\mathbb{R}^{N}$ equipped with the usual ordering and we fix the norm $\|X\|$ in $S(N)$ by setting $\|X\|=\sup \left\{|X \xi|\left|\xi \in \mathbb{R}^{N},\right| \xi \mid \leq 1\right\}=\sup \{|\lambda|: \lambda$ is an eigenvalue of $X\}$.

Let $\sigma: \mathbb{R}^{N} \rightarrow S(N)$ be the function defined by

$$
\sigma(p):=\frac{p \otimes p}{|p|^{2}}
$$

The $\infty$-Laplacian can be written as

$$
\Delta_{\infty} u=\operatorname{tr}\left(\sigma(D u) D^{2} u\right)
$$

for any $u \in C^{2}(\Omega)$.

It easy to check that $\sigma$ has the following properties:

- $\sigma(p)$ is homogeneous of order 0 , i.e., for any $\alpha \in \mathbb{R}$ and $p \in \mathbb{R}^{N}$

$$
\sigma(\alpha p)=\sigma(p)
$$

- for all $p \in \mathbb{R}^{N}$

where $I$ is the identity matrix in $\mathbb{R}^{N}$;

$$
0 \leq \sigma(p) \leq I
$$


- $\sigma(p)$ is idempotent, i.e.,

$$
(\sigma(p))^{2}=\sigma(p)
$$

- for any $p \in \mathbb{R}^{N} \backslash\{0\}$ and $p_{0} \in \mathbb{R}^{n}$ with $\left|p_{0}\right| \leq \frac{|p|}{2}$

$$
\operatorname{tr}\left[\left(\sigma\left(p+p_{0}\right)-\sigma(p)\right)^{2}\right] \leq 8 \frac{\left|p_{0}\right|^{2}}{|p|^{2}}
$$

The domain $\Omega$ is supposed to be bounded and of class $C^{2}$. In particular, it satisfies the interior sphere condition and the uniform exterior sphere condition, i.e.

$(\Omega 1)$ For each $x \in \partial \Omega$ there exist $R>0$ and $y \in \Omega$ for which $|x-y|=R$ and $B(y, R) \subset \Omega$.

$(\Omega 2)$ There exists $r>0$ such that $B(x+r \vec{n}(x), r) \cap \Omega=\emptyset$ for any $x \in \partial \Omega$.

From the property $(\Omega 2)$ it follows that

$$
\langle y-x, \vec{n}(x)\rangle \leq \frac{1}{2 r}|y-x|^{2} \quad \text { for } x \in \partial \Omega \text { and } y \in \bar{\Omega} .
$$

Moreover, the $C^{2}$-regularity of $\Omega$ implies the existence of a neighborhood of $\partial \Omega$ in $\bar{\Omega}$ on which the distance from the boundary

$$
d(x):=\inf \{|x-y|, y \in \partial \Omega\}, \quad x \in \bar{\Omega}
$$

is of class $C^{2}$. We still denote by $d$ a $C^{2}$ extension of the distance function to the whole $\bar{\Omega}$. Without loss of generality we can assume that $|D d(x)| \leq 1$ on $\bar{\Omega}$.

We adopt the notion of viscosity solution for (1.1) given in [4] for singular elliptic operators, in which is required to test only with test functions which have gradient different from zero.

We denote by $\operatorname{USC}(\bar{\Omega})$ the set of upper semicontinuous functions on $\bar{\Omega}$ and by $L S C(\bar{\Omega})$ the set of lower semicontinuous functions on $\bar{\Omega}$. Let $g: \bar{\Omega} \rightarrow \mathbb{R}$ and $B: \partial \Omega \times \mathbb{R} \times \mathbb{R}^{N} \rightarrow \mathbb{R}$.

Definition 2.1. Any function $u \in U S C(\bar{\Omega})$ (resp., $u \in L S C(\bar{\Omega})$ ) is called viscosity subsolution (resp., supersolution) of

$$
\begin{cases}\Delta_{\infty} u+b(x) \cdot D u+c(x) u=g(x) & \text { in } \Omega \\ B(x, u, D u)=0 & \text { on } \partial \Omega\end{cases}
$$

if the following conditions hold:

(i) For every $x_{0} \in \Omega$, for all $\varphi \in C^{2}(\bar{\Omega})$, such that $u-\varphi$ has a local maximum (resp., minimum) at $x_{0}$ and $D \varphi\left(x_{0}\right) \neq 0$, one has

$$
\Delta_{\infty} \varphi\left(x_{0}\right)+b\left(x_{0}\right) \cdot D \varphi\left(x_{0}\right)+c\left(x_{0}\right) u\left(x_{0}\right) \geq(\text { resp., } \leq) g\left(x_{0}\right)
$$

If $u \equiv k=$ const. in a neighborhood of $x_{0}$, then

$$
c\left(x_{0}\right) k \geq(\text { resp., } \leq) g\left(x_{0}\right)
$$

(ii) For every $x_{0} \in \partial \Omega$, for all $\varphi \in C^{2}(\bar{\Omega})$, such that $u-\varphi$ has a local maximum (resp., minimum) at $x_{0}$ and $D \varphi\left(x_{0}\right) \neq 0$, one has

$$
\left(-\Delta_{\infty} \varphi\left(x_{0}\right)-b\left(x_{0}\right) \cdot D \varphi\left(x_{0}\right)-c\left(x_{0}\right) u\left(x_{0}\right)+g\left(x_{0}\right)\right) \wedge B\left(x_{0}, u\left(x_{0}\right), D \varphi\left(x_{0}\right)\right) \leq 0
$$

(resp.,

$$
\left.\left(-\Delta_{\infty} \varphi\left(x_{0}\right)-b\left(x_{0}\right) \cdot D \varphi\left(x_{0}\right)-c\left(x_{0}\right) u\left(x_{0}\right)+g\left(x_{0}\right)\right) \vee B\left(x_{0}, u\left(x_{0}\right), D \varphi\left(x_{0}\right)\right) \geq 0\right) .
$$


If $u \equiv k=$ const. in a neighborhood of $x_{0}$ in $\bar{\Omega}$, then

$$
\left(-c\left(x_{0}\right) k+g\left(x_{0}\right)\right) \wedge B\left(x_{0}, k, 0\right) \leq 0
$$

(resp.,

$$
\left.\left(-c\left(x_{0}\right) k+g\left(x_{0}\right)\right) \vee B\left(x_{0}, k, 0\right) \geq 0\right) .
$$

It is possible to define sub and supersolutions of the $\infty$-Laplace equation also using the semicontinuous extensions of the function $(p, X) \rightarrow \operatorname{tr}(\sigma(p) X)$ as done in [11,12]. In Definition 2.1 it is remarkable that nothing is required in the case $D \varphi\left(x_{0}\right)=0$ if $u$ is not constant.

For a detailed presentation of the theory of viscosity solutions and of the boundary conditions in the viscosity sense, we refer the reader to e.g. [6].

We call strong viscosity subsolutions (resp., supersolutions) the viscosity subsolutions (resp., supersolutions) that satisfy $B(x, u, D u) \leq$ (resp., $\geq) 0$ in the viscosity sense for all $x \in \partial \Omega$. If $\lambda \rightarrow B(x, r, p-\lambda \vec{n})$ is nonincreasing in $\lambda \geq 0$, then classical subsolutions (resp., supersolutions) are strong viscosity subsolutions (resp., supersolutions), see [6], Proposition 7.2.

In the above definition the test functions can be substituted by the elements of the semijets $\bar{J}^{2,+} u\left(x_{0}\right)$ when $u$ is a subsolution and $\bar{J}^{2,-} u\left(x_{0}\right)$ when $u$ is a supersolution, see [6].

\section{LiPSCHITZ CONTINUITY OF VISCOSITY SOLUTIONS}

It is known that the $\infty$-harmonic functions, i.e., the solution of $\Delta_{\infty} u=0$ are locally Lipschitz continuous, see e.g. [2]. We now show the Lipschitz regularity in the whole $\bar{\Omega}$ of the solutions of the Neumann problem associated to the $\infty$-Laplacian plus lower order terms.

Theorem 3.1. Assume that $\Omega$ is a bounded domain of class $C^{2}$ and that $b, c, g$ are bounded in $\Omega$. If $u \in C(\bar{\Omega})$ is a viscosity solution of

then

$$
\begin{cases}\Delta_{\infty} u+b(x) \cdot D u+c(x) u=g(x) & \text { in } \Omega \\ \frac{\partial u}{\partial \vec{n}}=0 & \text { on } \partial \Omega,\end{cases}
$$

where $C_{0}$ depends on $\Omega, N,|b|_{\infty},|c|_{\infty},|g|_{\infty}$, and $|u|_{\infty}$.

The theorem is an immediate consequence of the next lemma, the proof of which, though following the line of Proposition III.1 of [9], introduces new test functions that, in particular, depend on the distance function $d(x)$.

The lemma will be used also in the proof of Theorem 4.8 in the next section.

Lemma 3.2. Assume the hypothesis of Theorem 3.1 and suppose that $g$ and $h$ are bounded functions. Let $u \in U S C(\bar{\Omega})$ be a viscosity subsolution of

$$
\begin{cases}\Delta_{\infty} u+b(x) \cdot D u+c(x) u=g(x) & \text { in } \Omega \\ \frac{\partial u}{\partial \bar{n}}=0 & \text { on } \partial \Omega\end{cases}
$$

and $v \in L S C(\bar{\Omega})$ a viscosity supersolution of

$$
\begin{cases}\Delta_{\infty} v+b(x) \cdot D v+c(x) v=h(x) & \text { in } \Omega \\ \frac{\partial v}{\partial \vec{n}}=0 & \text { on } \partial \Omega\end{cases}
$$

with $u$ and $v$ bounded, or $v \geq 0$ and bounded. If $m=\max _{\bar{\Omega}}(u-v) \geq 0$, then there exists $C_{0}>0$ such that

$$
u(x)-v(y) \leq m+C_{0}|x-y| \quad \forall x, y \in \bar{\Omega},
$$

where $C_{0}$ depends on $\Omega, N,|b|_{\infty},|c|_{\infty},|g|_{\infty},|h|_{\infty},|v|_{\infty}, m$ and $|u|_{\infty}$ or $\sup _{\bar{\Omega}} u$. 
Proof. We set

$$
\Phi(x)=M K|x|-M(K|x|)^{2},
$$

and

$$
\varphi(x, y)=m+\mathrm{e}^{-L(d(x)+d(y))} \Phi(x-y),
$$

where $L$ is a fixed number greater than $2 /(3 r)$ with $r$ the radius in the condition $(\Omega 2)$ and where $K$ and $M$ are two positive constants to be chosen later. If $K|x| \leq \frac{1}{4}$, then

$$
\Phi(x) \geq \frac{3}{4} M K|x| .
$$

We define

$$
\Delta_{K}:=\left\{(x, y) \in \mathbb{R}^{N} \times \mathbb{R}^{N}|| x-y \mid \leq \frac{1}{4 K}\right\}
$$

We fix $M$ such that

$$
\max _{\bar{\Omega}^{2}}(u(x)-v(y)) \leq m+\mathrm{e}^{-2 L d_{0}} \frac{M}{8},
$$

where $d_{0}=\max _{x \in \bar{\Omega}} d(x)$. To prove (3.1) it is enough to show that taking $K$ large enough, one has

$$
u(x)-v(y)-\varphi(x, y) \leq 0 \quad \text { for }(x, y) \in \Delta_{K} \cap \bar{\Omega}^{2} .
$$

Suppose by contradiction that for each $K$ there is some point $(\bar{x}, \bar{y}) \in \Delta_{K} \cap \bar{\Omega}^{2}$ such that

$$
u(\bar{x})-v(\bar{y})-\varphi(\bar{x}, \bar{y})=\max _{\Delta_{K} \cap \bar{\Omega}^{2}}(u(x)-v(y)-\varphi(x, y))>0 .
$$

Here we have dropped the dependence of $\bar{x}, \bar{y}$ on $K$ for simplicity of notations.

Observe that if $v \geq 0$, since from (3.2) $\Phi(x-y)$ is non-negative in $\Delta_{K}$ and $m \geq 0$, one has $u(\bar{x})>0$.

Clearly $\bar{x} \neq \bar{y}$. Moreover the point $(\bar{x}, \bar{y})$ belongs to $\operatorname{int}\left(\Delta_{K}\right) \cap \bar{\Omega}^{2}$. Indeed, if $|x-y|=\frac{1}{4 K}$, by (3.3) and (3.2) we have

$$
u(x)-v(y) \leq m+\mathrm{e}^{-2 L d_{0}} \frac{M}{8} \leq m+\mathrm{e}^{-L(d(x)+d(y))} \frac{1}{2} M K|x-y| \leq \varphi(x, y) .
$$

Since $\bar{x} \neq \bar{y}$ we can compute the derivatives of $\varphi$ at $(\bar{x}, \bar{y})$ obtaining

$$
\begin{aligned}
& D_{x} \varphi(\bar{x}, \bar{y})=\mathrm{e}^{-L(d(\bar{x})+d(\bar{y}))} M K\left\{-L|\bar{x}-\bar{y}|(1-K|\bar{x}-\bar{y}|) D d(\bar{x})+(1-2 K|\bar{x}-\bar{y}|) \frac{(\bar{x}-\bar{y})}{|\bar{x}-\bar{y}|}\right\}, \\
& D_{y} \varphi(\bar{x}, \bar{y})=\mathrm{e}^{-L(d(\bar{x})+d(\bar{y}))} M K\left\{-L|\bar{x}-\bar{y}|(1-K|\bar{x}-\bar{y}|) D d(\bar{y})-(1-2 K|\bar{x}-\bar{y}|) \frac{(\bar{x}-\bar{y})}{|\bar{x}-\bar{y}|}\right\} .
\end{aligned}
$$

Observe that for large $K$

$$
0<\mathrm{e}^{-L(d(\bar{x})+d(\bar{y}))} M K\left(\frac{1}{2}-L|\bar{x}-\bar{y}|\right) \leq\left|D_{x} \varphi(\bar{x}, \bar{y})\right|,\left|D_{y} \varphi(\bar{x}, \bar{y})\right| \leq 2 M K .
$$


Using (2.2), if $\bar{x} \in \partial \Omega$ we have

$$
\begin{aligned}
\left\langle D_{x} \varphi(\bar{x}, \bar{y}), \vec{n}(\bar{x})\right\rangle & =\mathrm{e}^{-L d(\bar{y})} M K\left\{L|\bar{x}-\bar{y}|(1-K|\bar{x}-\bar{y}|)+(1-2 K|\bar{x}-\bar{y}|)\left\langle\frac{(\bar{x}-\bar{y})}{|\bar{x}-\bar{y}|}, \vec{n}(\bar{x})\right\rangle\right\} \\
& \geq \mathrm{e}^{-L d(\bar{y})} M K\left\{\frac{3}{4} L|\bar{x}-\bar{y}|-(1-2 K|\bar{x}-\bar{y}|) \frac{|\bar{x}-\bar{y}|}{2 r}\right\} \\
& \geq \frac{1}{2} \mathrm{e}^{-L d(\bar{y})} M K|\bar{x}-\bar{y}|\left(\frac{3}{2} L-\frac{1}{r}\right)>0
\end{aligned}
$$

since $\bar{x} \neq \bar{y}$ and $L>2 /(3 r)$. Similarly, if $\bar{y} \in \partial \Omega$

$$
\left\langle-D_{y} \varphi(\bar{x}, \bar{y}), \vec{n}(\bar{y})\right\rangle \leq \frac{1}{2} \mathrm{e}^{-L d(\bar{x})} M K|\bar{x}-\bar{y}|\left(-\frac{3}{2} L+\frac{1}{r}\right)<0 .
$$

In view of definition of sub and supersolution, we conclude that

$$
\begin{aligned}
\operatorname{tr}\left(\sigma\left(D_{x} \varphi(\bar{x}, \bar{y})\right) X\right)+b(\bar{x}) \cdot D_{x} \varphi(\bar{x}, \bar{y})+c(\bar{x}) u(\bar{x}) \geq g(\bar{x}) & \text { if }\left(D_{x} \varphi(\bar{x}, \bar{y}), X\right) \in \bar{J}^{2,+} u(\bar{x}), \\
\operatorname{tr}\left(\sigma\left(D_{y} \varphi(\bar{x}, \bar{y})\right) Y\right)-b(\bar{y}) \cdot D_{y} \varphi(\bar{x}, \bar{y})+c(\bar{y}) v(\bar{y}) \leq h(\bar{y}) & \text { if }\left(-D_{y} \varphi(\bar{x}, \bar{y}), Y\right) \in \bar{J}^{2,-} v(\bar{y}) .
\end{aligned}
$$

Then the previous inequalities holds for any maximum point $(\bar{x}, \bar{y}) \in \Delta_{K} \cap \bar{\Omega}^{2}$, provided $K$ is large enough.

Since $(\bar{x}, \bar{y}) \in \operatorname{int} \Delta_{K} \cap \bar{\Omega}^{2}$, it is a local maximum of $u(x)-v(y)-\varphi(x, y)$ in $\bar{\Omega}^{2}$. Applying Theorem 3.2 in [6], for every $\epsilon>0$ there exist $X, Y \in S(N)$ such that $\left(D_{x} \varphi(\bar{x}, \bar{y}), X\right) \in \bar{J}^{2,+} u(\bar{x}),\left(-D_{y} \varphi(\bar{x}, \bar{y}), Y\right) \in \bar{J}^{2,-} v(\bar{y})$ and

$$
\left(\begin{array}{cc}
X & 0 \\
0 & -Y
\end{array}\right) \leq D^{2}(\varphi(\bar{x}, \bar{y}))+\epsilon\left(D^{2}(\varphi(\bar{x}, \bar{y}))\right)^{2}
$$

Now we want to estimate the matrix on the right-hand side of the last inequality:

$$
\begin{aligned}
D^{2} \varphi(\bar{x}, \bar{y})= & \Phi(\bar{x}-\bar{y}) D^{2}\left(\mathrm{e}^{-L(d(\bar{x})+d(\bar{y}))}\right)+D\left(\mathrm{e}^{-L(d(\bar{x})+d(\bar{y}))}\right) \otimes D(\Phi(\bar{x}-\bar{y})) \\
& +D(\Phi(\bar{x}-\bar{y})) \otimes D\left(\mathrm{e}^{-L(d(\bar{x})+d(\bar{y}))}\right)+\mathrm{e}^{-L(d(\bar{x})+d(\bar{y}))} D^{2}(\Phi(\bar{x}-\bar{y})) .
\end{aligned}
$$

We set

$$
\begin{aligned}
& A_{1}:=\Phi(\bar{x}-\bar{y}) D^{2}\left(\mathrm{e}^{-L(d(\bar{x})+d(\bar{y}))}\right), \\
& A_{2}:=D\left(\mathrm{e}^{-L(d(\bar{x})+d(\bar{y}))}\right) \otimes D(\Phi(\bar{x}-\bar{y}))+D(\Phi(\bar{x}-\bar{y})) \otimes D\left(\mathrm{e}^{-L(d(\bar{x})+d(\bar{y}))}\right), \\
& A_{3}:=\mathrm{e}^{-L(d(\bar{x})+d(\bar{y}))} D^{2}(\Phi(\bar{x}-\bar{y})) .
\end{aligned}
$$

Observe that

$$
A_{1} \leq C K|\bar{x}-\bar{y}|\left(\begin{array}{cc}
I & 0 \\
0 & I
\end{array}\right)
$$

Here and henceforth $C$ denotes various positive constants independent of $K$.

For $A_{2}$ we have the following estimate

$$
A_{2} \leq C K\left(\begin{array}{cc}
I & 0 \\
0 & I
\end{array}\right)+C K\left(\begin{array}{cc}
I & -I \\
-I & I
\end{array}\right) .
$$


Indeed for $\xi, \eta \in \mathbb{R}^{N}$ we compute

$$
\begin{aligned}
\left\langle A_{2}(\xi, \eta),(\xi, \eta)\right\rangle= & 2 L \mathrm{e}^{-L(d(\bar{x})+d(\bar{y}))}\{\langle D d(\bar{x}) \otimes D \Phi(\bar{x}-\bar{y})(\eta-\xi), \xi\rangle \\
& +\langle D d(\bar{y}) \otimes D \Phi(\bar{x}-\bar{y})(\eta-\xi), \eta\rangle\} \leq C K(|\xi|+|\eta|)|\eta-\xi| \\
\leq & C K\left(|\xi|^{2}+|\eta|^{2}\right)+C K|\eta-\xi|^{2} .
\end{aligned}
$$

Now we consider $A_{3}$. The matrix $D^{2}(\Phi(\bar{x}-\bar{y}))$ has the form

$$
D^{2}(\Phi(\bar{x}-\bar{y}))=\left(\begin{array}{cc}
D^{2} \Phi(\bar{x}-\bar{y}) & -D^{2} \Phi(\bar{x}-\bar{y}) \\
-D^{2} \Phi(\bar{x}-\bar{y}) & D^{2} \Phi(\bar{x}-\bar{y})
\end{array}\right)
$$

and the Hessian matrix of $\Phi(x)$ is

$$
D^{2} \Phi(x)=\frac{M K}{|x|}\left(I-\frac{x \otimes x}{|x|^{2}}\right)-2 M K^{2} I .
$$

If we choose

$$
\epsilon=\frac{|\bar{x}-\bar{y}|}{2 M K \mathrm{e}^{-L(d(\bar{x})+d(\bar{y}))}},
$$

then we have the following estimates

$$
\begin{gathered}
\epsilon A_{1}^{2} \leq C K|\bar{x}-\bar{y}|^{3} I_{2 N}, \quad \epsilon A_{2}^{2} \leq C K|\bar{x}-\bar{y}| I_{2 N}, \\
\epsilon\left(A_{1} A_{2}+A_{2} A_{1}\right) \leq C K|\bar{x}-\bar{y}|^{2} I_{2 N}, \\
\epsilon\left(A_{1} A_{3}+A_{3} A_{1}\right) \leq C K|\bar{x}-\bar{y}| I_{2 N}, \quad \epsilon\left(A_{2} A_{3}+A_{3} A_{2}\right) \leq C K I_{2 N},
\end{gathered}
$$

where $I_{2 N}:=\left(\begin{array}{cc}I & 0 \\ 0 & I\end{array}\right)$. Then using (3.6), (3.7), (3.10) and observing that

$$
\left(D^{2}(\Phi(\bar{x}-\bar{y}))\right)^{2}=\left(\begin{array}{cc}
2\left(D^{2} \Phi(\bar{x}-\bar{y})\right)^{2} & -2\left(D^{2} \Phi(\bar{x}-\bar{y})\right)^{2} \\
-2\left(D^{2} \Phi(\bar{x}-\bar{y})\right)^{2} & 2\left(D^{2} \Phi(\bar{x}-\bar{y})\right)^{2}
\end{array}\right),
$$

from (3.5) we conclude that

$$
\left(\begin{array}{cc}
X & 0 \\
0 & -Y
\end{array}\right) \leq O(K)\left(\begin{array}{cc}
I & 0 \\
0 & I
\end{array}\right)+\left(\begin{array}{cc}
B & -B \\
-B & B
\end{array}\right)
$$

where

$$
B=C K I+\mathrm{e}^{-L(d(\bar{x})+d(\bar{y}))}\left[D^{2} \Phi(\bar{x}-\bar{y})+\frac{|\bar{x}-\bar{y}|}{M K}\left(D^{2} \Phi(\bar{x}-\bar{y})\right)^{2}\right] .
$$

The last inequality can be rewritten as follows

$$
\left(\begin{array}{cc}
\widetilde{X} & 0 \\
0 & -\widetilde{Y}
\end{array}\right) \leq\left(\begin{array}{cc}
B & -B \\
-B & B
\end{array}\right),
$$

with $\widetilde{X}=X-O(K) I$ and $\widetilde{Y}=Y+O(K) I$. Multiplying on the left the previous inequality by the non-negative symmetric matrix

$$
\left(\begin{array}{cc}
\sigma\left(D_{x} \varphi(\bar{x}, \bar{y})\right) & 0 \\
0 & \sigma\left(D_{y} \varphi(\bar{x}, \bar{y})\right)
\end{array}\right)
$$


and taking traces we get

$$
\operatorname{tr}\left(\sigma\left(D_{x} \varphi(\bar{x}, \bar{y})\right) \tilde{X}\right)-\operatorname{tr}\left(\sigma\left(D_{y} \varphi(\bar{x}, \bar{y})\right) \widetilde{Y}\right) \leq \operatorname{tr}\left(\sigma\left(D_{x} \varphi(\bar{x}, \bar{y})\right) B\right)+\operatorname{tr}\left(\sigma\left(D_{y} \varphi(\bar{x}, \bar{y})\right) B\right) .
$$

We want to get a good estimate for the matrix on the right-hand side above. For that aim let

$$
0 \leq P:=\frac{(\bar{x}-\bar{y}) \otimes(\bar{x}-\bar{y})}{|\bar{x}-\bar{y}|^{2}} \leq I,
$$

and let us compute $\operatorname{tr}(P B)$. From (3.8), since the matrix $\left(1 /|x|^{2}\right) x \otimes x$ is idempotent, we get

$$
\left(D^{2} \Phi(x)\right)^{2}=\frac{M^{2} K^{2}}{|x|^{2}}(1-4 K|x|)\left(I-\frac{x \otimes x}{|x|^{2}}\right)+4 M^{2} K^{4} I .
$$

Then, using that $\operatorname{tr} P=1$ and $4 K|\bar{x}-\bar{y}| \leq 1$, we have

$$
\begin{aligned}
\operatorname{tr}(P B) & =C K+\mathrm{e}^{-L(d(\bar{x})+d(\bar{y}))}\left(-2 M K^{2}+4 M K^{3}|\bar{x}-\bar{y}|\right) \\
& \leq C K-\mathrm{e}^{-L(d(\bar{x})+d(\bar{y}))} M K^{2} \leq-C K^{2},
\end{aligned}
$$

for large $K$. The vector $D_{x} \varphi(\bar{x}, \bar{y})$ can be written in the following way

$$
D_{x} \varphi(\bar{x}, \bar{y})=\mathrm{e}^{-L(d(\bar{x})+d(\bar{y}))} M K\left(v_{1}+v_{2}\right)
$$

where

and so

$$
v_{1}=-L|\bar{x}-\bar{y}|(1-K|\bar{x}-\bar{y}|) D d(\bar{x}), \quad v_{2}=(1-2 K|\bar{x}-\bar{y}|) \frac{(\bar{x}-\bar{y})}{|\bar{x}-\bar{y}|}
$$

$$
\sigma\left(D_{x} \varphi(\bar{x}, \bar{y})\right)=\frac{v_{1} \otimes v_{1}}{\left|v_{1}+v_{2}\right|^{2}}+\frac{v_{1} \otimes v_{2}+v_{2} \otimes v_{1}}{\left|v_{1}+v_{2}\right|^{2}}+\frac{v_{2} \otimes v_{2}}{\left|v_{1}+v_{2}\right|^{2}} .
$$

Since $K|\bar{x}-\bar{y}| \leq \frac{1}{4}$, for large $K$ we have

$$
\frac{1}{4}=\frac{1}{2}-\frac{1}{4} \leq\left|v_{2}\right|-\left|v_{1}\right| \leq\left|v_{1}+v_{2}\right| \leq\left|v_{1}\right|+\left|v_{2}\right| \leq 2,
$$

and

Then

$$
\|B\| \leq \frac{C K}{|\bar{x}-\bar{y}|}
$$

and

$$
\begin{aligned}
& \left|\operatorname{tr}\left(\frac{v_{1} \otimes v_{1}}{\left|v_{1}+v_{2}\right|^{2}} B\right)\right| \leq C|\bar{x}-\bar{y}|^{2}\|B\| \leq C K|\bar{x}-\bar{y}|, \\
& \left|\operatorname{tr}\left(\frac{v_{1} \otimes v_{2}+v_{2} \otimes v_{1}}{\left|v_{1}+v_{2}\right|^{2}} B\right)\right| \leq C|\bar{x}-\bar{y}|\|B\| \leq C K
\end{aligned}
$$

In conclusion

$$
\operatorname{tr}\left(\frac{v_{2} \otimes v_{2}}{\left|v_{1}+v_{2}\right|^{2}} B\right)=\frac{1}{\left|v_{1}+v_{2}\right|^{2}} \operatorname{tr}(P B) \leq-C K^{2} .
$$

$$
\operatorname{tr}\left(\sigma\left(D_{x} \varphi(\bar{x}, \bar{y}) B\right)\right) \leq O(K)-C K^{2} .
$$

The same estimate holds for $\operatorname{tr}\left(\sigma\left(D_{y} \varphi(\bar{x}, \bar{y})\right) B\right)$. Hence, from (3.11) we conclude that

$$
\operatorname{tr}\left(\sigma\left(D_{x} \varphi(\bar{x}, \bar{y})\right) \tilde{X}\right)-\operatorname{tr}\left(\sigma\left(D_{y} \varphi(\bar{x}, \bar{y})\right) \tilde{Y}\right) \leq O(K)-C K^{2}
$$


Now, using the previous estimate, the definition of $\widetilde{X}$ and $\widetilde{Y}$ and the fact that $u$ and $v$ are respectively suband supersolution we compute

$$
\begin{aligned}
g(\bar{x})-c(\bar{x}) u(\bar{x}) & \leq \operatorname{tr}\left(\sigma\left(D_{x} \varphi\right) X\right)+b(\bar{x}) \cdot D_{x} \varphi \\
& \leq \operatorname{tr}\left(\sigma\left(D_{x} \varphi\right) \tilde{X}\right)+O(K)+b(\bar{x}) \cdot D_{x} \varphi \\
& \leq \operatorname{tr}\left(\sigma\left(D_{y} \varphi\right) Y\right)+O(K)-C K^{2}+b(\bar{x}) \cdot D_{x} \varphi \\
& \leq b(\bar{y}) \cdot D_{y} \varphi-c(\bar{y}) v(\bar{y})+h(\bar{y})+O(K)-C K^{2}+b(\bar{x}) \cdot D_{x} \varphi .
\end{aligned}
$$

From this inequalities, using (3.4) we get

$$
g(\bar{x})-h(\bar{y})-c(\bar{x}) u(\bar{x})+c(\bar{y}) v(\bar{y}) \leq O(K)-C K^{2} .
$$

If both $u$ and $v$ are bounded, then the member on the left-hand side of the last inequality is bounded from below by $-|g|_{\infty}-|h|_{\infty}-|c|_{\infty}\left(|u|_{\infty}+|v|_{\infty}\right)$. Otherwise, if $v$ is non-negative and bounded, then $u(\bar{x}) \geq 0$ and that quantity is greater than $-|g|_{\infty}-|h|_{\infty}-|c|_{\infty}\left(\sup u+|v|_{\infty}\right)$. On the other hand, the member on the right-hand side goes to $-\infty$ as $K \rightarrow+\infty$, hence taking $K$ large enough we obtain a contradiction and this concludes the proof.

Remark 3.3. If $u$ is a subsolution of $\Delta_{\infty} u+b(x) \cdot D u+c(x) u=g, v$ is a supersolution of $\Delta_{\infty} v+b(x) \cdot D v+$ $c(x) v=h$ in $\Omega, u \leq v$ on $\partial \Omega$ and $m>0$ then the estimate (3.1) still holds for any $x, y \in \Omega$. To prove this define $\varphi=m+M K|x|-M(K|x|)^{2}$ and follow the proof of Lemma 3.2.

Since the Lipschitz estimate depends only on the bounds of the solution of $g$ and on the structural constants, an immediate consequence of Theorem 3.1 is the following compactness criterion that will be useful in the next sections.

Corollary 3.4. Assume the hypothesis of Theorem 3.1 on $\Omega, F$ and b. Suppose that $\left(g_{n}\right)_{n}$ is a sequence of continuous and uniformly bounded functions and $\left(u_{n}\right)_{n}$ is a sequence of uniformly bounded viscosity solutions of

$$
\begin{cases}\Delta_{\infty} u_{n}+b(x) \cdot D u_{n}=g_{n}(x) & \text { in } \Omega \\ \frac{\partial u_{n}}{\partial \bar{n}^{2}}=0 & \text { on } \partial \Omega .\end{cases}
$$

Then the sequence $\left(u_{n}\right)_{n}$ is relatively compact in $C(\bar{\Omega})$.

\section{The Maximum PRINCIPle AND the PRINCIPAl EIGENVALUes}

We say that the operator $\Delta_{\infty}+b(x) \cdot D+c(x)$ with the Neumann boundary condition satisfies the maximum principle if whenever $u \in U S C(\bar{\Omega})$ is a viscosity subsolution of

$$
\begin{cases}\Delta_{\infty} u+b(x) \cdot D u+c(x) u=0 & \text { in } \Omega \\ \frac{\partial u}{\partial \vec{n}}=0 & \text { on } \partial \Omega,\end{cases}
$$

then $u \leq 0$ on $\bar{\Omega}$.

We first prove that the maximum principle holds under the classical assumption $c \leq 0$, also for domain which are not of class $C^{2}$ and with more general boundary conditions. Then we show that the operator $\Delta_{\infty}+b(x) \cdot D+c(x)+\lambda$ with the Neumann boundary condition satisfies the maximum principle for any $\lambda<\bar{\lambda}$. This is the best result that one can expect, indeed, as we will see, $\bar{\lambda}$ admits a positive eigenfunction which provides a counterexample to the maximum principle for $\lambda \geq \bar{\lambda}$.

Finally, we give an example of class of functions $c(x)$ which change sign in $\Omega$ and such that the associated principal eigenvalue $\bar{\lambda}$ is positive. 


\subsection{The case $c(x) \leq 0$}

In this subsection we assume that $\Omega$ is of class $C^{1}$ and satisfies the interior sphere condition $(\Omega 1)$. We need the comparison principle between sub- and supersolutions of the Dirichlet problem when $c<0$ in $\Omega$. This result is known for the operator $\Delta_{\infty} u+b(x) \cdot D u+c(x) u$ when $b$ is Lipschitz continuous or $b$ satisfies $\langle b(x)-b(y), x-y\rangle \leq 0$, see e.g. [6]. Actually, we can remove these conditions.

Theorem 4.1. Let $\Omega$ be bounded. Assume that b, $c$ and $g$ are continuous and bounded in $\Omega$ and $c<0$ on $\bar{\Omega}$. If $u \in U S C(\bar{\Omega})$ and $v \in L S C(\bar{\Omega})$ are respectively sub- and supersolution of

$$
\Delta_{\infty} u+b(x) \cdot D u+c(x) u=g(x) \quad \text { in } \Omega
$$

and $u \leq v$ on $\partial \Omega$ then $u \leq v$ in $\Omega$.

For convenience of the reader the proof of the theorem will be sketched at the end of the next subsection.

The previous comparison result allows us to establish the strong minimum and maximum principles, for suband supersolutions of the Neumann problem even with the following more general boundary condition

$$
f(x, u)+\frac{\partial u}{\partial \vec{n}}=0 \quad x \in \partial \Omega
$$

for some $f: \partial \Omega \times \mathbb{R} \rightarrow \mathbb{R}$.

Proposition 4.2. Let $\Omega$ be a $C^{1}$ domain satisfying $(\Omega 1)$. Suppose that $b$ and $c$ are bounded and continuous in $\Omega$ and that $f(x, 0) \leq 0$ for all $x \in \partial \Omega$. If $v \in L S C(\bar{\Omega})$ is a non-negative viscosity supersolution of

$$
\begin{cases}\Delta_{\infty} v+b(x) \cdot D v+c(x) v=0 & \text { in } \Omega \\ f(x, v)+\frac{\partial v}{\partial \vec{n}}=0 & \text { on } \partial \Omega\end{cases}
$$

then either $v \equiv 0$ or $v>0$ on $\bar{\Omega}$.

Proof. Since $v$ is non-negative, it is supersolution in $\Omega$ of the equation

$$
\Delta_{\infty} v+b(x) \cdot D v-|c|_{\infty} v=0
$$

Without loss of generality we can assume $|c|_{\infty}>0$. Suppose by contradiction that $v \not \equiv 0$ vanishes somewhere in $\Omega$. Then we can find $x_{1}, x_{0} \in \Omega$ and $R>0$ such that $B\left(x_{1}, \frac{3}{2} R\right) \subset \Omega, v>0$ in $B\left(x_{1}, R\right),\left|x_{1}-x_{0}\right|=R$ and $v\left(x_{0}\right)=0$. Let us construct a subsolution of (4.2) in the annulus $\frac{R}{2}<\left|x-x_{1}\right|=r<\frac{3}{2} R$.

Let us consider the function $\phi(x)=\mathrm{e}^{-k r}-\mathrm{e}^{-k R}$, where $k$ is a positive constant to be determined. It easy to see that for radial functions $g(x)=\varphi(r), \Delta_{\infty} g(x)=\varphi^{\prime \prime}(r)$. Then

$$
\begin{aligned}
\Delta_{\infty} \phi+b(x) \cdot D \phi-|c|_{\infty} \phi & =k^{2} \mathrm{e}^{-k r}-k \mathrm{e}^{-k r} b(x) \cdot \frac{(x-y)}{r}-|c|_{\infty}\left(\mathrm{e}^{-k r}-\mathrm{e}^{-k R}\right) \\
& \geq \mathrm{e}^{-k r}\left(k^{2}-|b|_{\infty} k-|c|_{\infty}\right) .
\end{aligned}
$$

Take $k$ such that

$$
k^{2}-|b|_{\infty} k-|c|_{\infty}>0
$$

then $\phi$ is a strict subsolution of the equation (4.2). Now choose $m>0$ such that

$$
m\left(\mathrm{e}^{-k \frac{R}{2}}-\mathrm{e}^{-k R}\right)=v_{1}:=\inf _{\left|x-x_{1}\right|=\frac{R}{2}} v(x)>0,
$$

and define $w(x)=m\left(\mathrm{e}^{-k r}-\mathrm{e}^{-k R}\right)$. By homogeneity $w$ is still a subsolution of (4.2) in the annulus $\frac{R}{2}<$ $\left|x-x_{1}\right|<\frac{3}{2} R$, moreover $w=v_{1} \leq v$ if $\left|x-x_{1}\right|=\frac{R}{2}$ and $w<0 \leq v$ if $\left|x-x_{1}\right|=\frac{3}{2} R$. Then by the comparison principle, Theorem 4.1, $w \leq v$ in the entire annulus. 
Since $v\left(x_{0}\right)=w\left(x_{0}\right)=0, w$ is a test function for $v$ at $x_{0}$ with $D w\left(x_{0}\right) \neq 0$. But

$$
\Delta_{\infty} w\left(x_{0}\right)+b\left(x_{0}\right) \cdot D w\left(x_{0}\right)-|c|_{\infty} v\left(x_{0}\right)>0,
$$

and this contradicts the definition of $v$. Then $v>0$ in $\Omega$.

Now suppose by contradiction that $x_{0}$ is some point in $\partial \Omega$ on which $v\left(x_{0}\right)=0$. The interior sphere condition $(\Omega 1)$ implies that there exist $R>0$ and $y \in \Omega$ such that the ball centered in $y$ and of radius $R, B(y, R)$, is contained in $\Omega$ and $x_{0} \in \partial B(y, R)$. Fixed $0<\rho<R$, as before the function $w(x)=m\left(\mathrm{e}^{-k r}-\mathrm{e}^{-k R}\right)$ is a strict subsolution of (4.2) in the annulus $\rho<|x-y|=r<R$, where $m$ is such that $m\left(\mathrm{e}^{-k \rho}-\mathrm{e}^{-k R}\right)=$ $v_{1}:=\inf _{|x-y|=\rho} v(x)>0$. Since $w \leq v$ on the boundary of the annulus then again by the comparison principle, Theorem $4.1, w \leq v$ in the entire annulus.

Now let $\delta$ be a positive number smaller than $R-\rho$. In $B\left(x_{0}, \delta\right) \cap \bar{\Omega}$ still $w \leq v$, since for $|x-y|>R$, $w<0 \leq v$; moreover $w\left(x_{0}\right)=v\left(x_{0}\right)=0$. Then $w$ is a test function for $v$ at $x_{0}$. But

$$
\Delta_{\infty} w\left(x_{0}\right)+b\left(x_{0}\right) \cdot D w\left(x_{0}\right)-|c|_{\infty} v\left(x_{0}\right)>0,
$$

and

$$
f\left(x_{0}, v\left(x_{0}\right)\right)+\frac{\partial w}{\partial \vec{n}}\left(x_{0}\right)=f\left(x_{0}, 0\right)-k m \mathrm{e}^{-k R}<0 .
$$

This contradicts the definition of $v$. Finally $v$ cannot be zero on $\bar{\Omega}$.

Similarly we can prove:

Proposition 4.3. Let $\Omega$ be a $C^{1}$ domain satisfying $(\Omega 1)$. Assume that $b$ and $c$ are bounded and continuous in $\Omega$ and that $f(x, 0) \geq 0$ for all $x \in \partial \Omega$. If $u \in U S C(\bar{\Omega})$ is a non-positive viscosity subsolution of (4.1) then either $u \equiv 0$ or $u<0$ on $\bar{\Omega}$.

For $x \in \partial \Omega$, let us introduce $S(x)$, the symmetric operator corresponding to the second fundamental form of $\partial \Omega$ in $x$ oriented with the exterior normal to $\Omega$.

Theorem 4.4 (maximum principle for $c \leq 0$ ). Assume the hypothesis of Proposition 4.3. In addition suppose that $\Omega$ is bounded, $c \leq 0, c \neq \equiv$ and $r \rightarrow f(x, r)$ is non-decreasing on $\mathbb{R}$. If $u \in U S C(\bar{\Omega})$ is a viscosity subsolution of (4.1) then $u \leq 0$ on $\bar{\Omega}$. The same conclusion holds also if $c \equiv 0$ in the following two cases:

(i) $\Omega$ is a $C^{2}$ domain and for any $r>0$ there exists $\bar{x} \in \partial \Omega$ such that $f(\bar{x}, r)>0, S(\bar{x}) \leq 0$ and $\langle b(\bar{x}), \vec{n}(\bar{x})\rangle>0$;

(ii) $\max _{x \in \partial \Omega} f(x, r)>0$ for any $r>0$ and $u$ is a strong subsolution.

Proof. Let $u$ be a subsolution of (4.1) and $c \not \equiv 0$. First let us suppose $u \equiv k=$ const. By definition

$$
c(x) k \geq 0 \quad \text { in } \Omega,
$$

which implies $k \leq 0$.

Now we assume that $u$ is not a constant. We argue by contradiction; suppose that $\max _{\bar{\Omega}} u=u\left(x_{0}\right)>0$, for some $x_{0} \in \bar{\Omega}$. Define $\widetilde{u}(x):=u(x)-u\left(x_{0}\right)$. Since $c \leq 0$ and $f$ is non-decreasing, $\widetilde{u}$ is a non-positive subsolution of (4.1). Then, from Proposition 4.3, either $u \equiv u\left(x_{0}\right)$ or $u<u\left(x_{0}\right)$ on $\bar{\Omega}$. In both cases we get a contradiction.

Let us turn to the case $c \equiv 0$. We have to prove that $u$ cannot be a positive constant. Suppose by contradiction that $u \equiv k$. Suppose that $\Omega$ is a $C^{2}$ domain and let $\bar{x} \in \partial \Omega$ be such that $S(\bar{x}) \leq 0,\langle b(\bar{x}), \vec{n}(\bar{x})\rangle>0$ and $f(\bar{x}, k)>0$. In general, if $\phi$ is a $C^{2}$ function, $\bar{x} \in \partial \Omega$ and $S(\bar{x}) \leq 0$, then $\left(D \phi(\bar{x})-\lambda \vec{n}(\bar{x}), D^{2} \phi(\bar{x})\right) \in J^{2,+} \phi(\bar{x})$, for $\lambda \geq 0$ (see [6], Rem. 2.7). Hence $(-\lambda \vec{n}(\bar{x}), 0) \in J^{2,+} u(\bar{x})$. But

$$
f(\bar{x}, k)-\lambda\langle\vec{n}(\bar{x}), \vec{n}(\bar{x})\rangle=f(\bar{x}, k)-\lambda>0,
$$

for $\lambda>0$ small enough, and

This contradicts the definition of $u$.

$$
-\lambda\langle b(\bar{x}), \vec{n}(\bar{x})\rangle<0
$$


Finally if $u$ is a strong subsolution, $u \equiv k>0$ and $f(\bar{x}, k)>0$ for some $\bar{x} \in \partial \Omega$, then the boundary condition is not satisfied at $\bar{x}$ for $p=0$.

Remark 4.5. Under the same assumptions of Theorem 4.4, but now with $f$ satisfying $f(x, 0) \leq 0$ for all $x \in \partial \Omega$ and with $f(\bar{x}, r)<0$ for $r<0$ in (i) and $\min _{x \in \partial \Omega} f(x, r)<0$ for $r<0$ in (ii), using Proposition 4.2 we can prove the minimum principle, i.e., if $u \in L S C(\bar{\Omega})$ is a viscosity supersolution of (4.1) then $u \geq 0$ on $\bar{\Omega}$.

Remark 4.6. $C^{2}$ convex sets satisfy the condition $S \leq 0$ in every point of the boundary.

Remark 4.7. If $c \equiv 0$ and $f \equiv 0$ a counterexample to the maximum principle is given by the positive constants.

\subsection{The threshold for the maximum principle}

In this subsection and in the rest of the paper we always assume that $\Omega$ is bounded and of class $C^{2}$ and that $b$ and $c$ are continuous on $\bar{\Omega}$.

Theorem 4.8 (maximum principle for $\lambda<\bar{\lambda}$ ). Let $\lambda<\bar{\lambda}$ and let $u \in U S C(\bar{\Omega})$ be a viscosity subsolution of

$$
\begin{cases}\Delta_{\infty} u+b(x) \cdot D u+(c(x)+\lambda) u=0 & \text { in } \Omega \\ \frac{\partial u}{\partial \vec{n}}=0 & \text { on } \partial \Omega\end{cases}
$$

then $u \leq 0$ on $\bar{\Omega}$.

Corollary 4.9. The quantity $\bar{\lambda}$ is finite.

Proof. It suffices to observe that $\bar{\lambda} \leq|c|_{\infty}$, since when the zero order coefficient is $c(x)+|c|_{\infty}$ the maximum principle does not hold. A counterexample is given by the positive constants.

In the proof of Theorem 4.8 we need the following result which is an adaptation of Lemma 1 of [4] for supersolutions of the Neumann boundary value problem.

Lemma 4.10. Let $v \in L S C(\bar{\Omega})$ be a viscosity supersolution of

$$
\begin{cases}\Delta_{\infty} v+b(x) \cdot D v-\beta(v(x))=g(x) & \text { in } \Omega \\ \frac{\partial v}{\partial \vec{n}}=0 & \text { on } \partial \Omega,\end{cases}
$$

for some functions $g, \beta \in U S C(\bar{\Omega})$. Suppose that $\bar{x} \in \bar{\Omega}$ is a strict local minimum of $v(x)+C|x-\bar{x}|^{q} \mathrm{e}^{-k d(x)}$, $k>\frac{q}{2 r}$, where $r$ is the radius in the condition $(\Omega 2)$ and $q>2$. Moreover suppose that $v$ is not locally constant around $\bar{x}$. Then

$$
-\beta(v(\bar{x})) \leq g(\bar{x}) .
$$

Remark 4.11. Similarly, if $\beta, g \in L S C(\bar{\Omega}), u \in U S C(\bar{\Omega})$ is a supersolution, $\bar{x}$ is a strict local maximum of $u(x)-C|x-\bar{x}|^{q} \mathrm{e}^{-k d(x)}, k>\frac{q}{2 r}, q>2$ and $u$ is not locally constant around $\bar{x}$, it can be proved that

$$
-\beta(u(\bar{x})) \geq g(\bar{x}) .
$$

Proof of Theorem 4.8. Let $\tau \in] \lambda, \bar{\lambda}[$, then by definition there exists $v>0$ on $\bar{\Omega}$ bounded viscosity supersolution of

$$
\begin{cases}\Delta_{\infty} v+b(x) \cdot D v+(c(x)+\tau) v=0 & \text { in } \Omega \\ \frac{\partial v}{\partial \vec{n}}=0 & \text { on } \partial \Omega .\end{cases}
$$

We argue by contradiction and suppose that $u$ has a positive maximum in $\bar{\Omega}$. As in [4], we define $\gamma^{\prime}:=$ $\sup _{\bar{\Omega}}(u / v)>0$ and $w=\gamma v$, with $\gamma \in\left(0, \gamma^{\prime}\right)$ to be determined. By homogeneity, $w$ is still a supersolution 
of (4.4). Let $\bar{y} \in \bar{\Omega}$ be such that $u(\bar{y}) / v(\bar{y})=\gamma^{\prime}$. Since $u(\bar{y})-w(\bar{y})=\left(\gamma^{\prime}-\gamma\right) v(\bar{y})>0$, the supremum of $u-w$ is strictly positive, then by upper semicontinuity there exists $\bar{x} \in \bar{\Omega}$ such that

$$
u(\bar{x})-w(\bar{x})=\max _{\bar{\Omega}}(u-w)=m>0 .
$$

Clearly $u(\bar{x})>w(\bar{x})>0$, moreover $u(\bar{x}) \leq \gamma^{\prime} v(\bar{x})=\frac{\gamma^{\prime}}{\gamma} w(\bar{x})$, from which

$$
w(\bar{x}) \geq \frac{\gamma}{\gamma^{\prime}} u(\bar{x}) .
$$

Fix $q>2$ and $k>q /(2 r)$, where $r$ is the radius in the condition ( $\Omega 2)$, and define for $j \in \mathbb{N}$ the functions $\phi \in C^{2}(\bar{\Omega} \times \bar{\Omega})$ and $\psi \in U S C(\bar{\Omega} \times \bar{\Omega})$ by

$$
\phi(x, y)=\frac{j}{q}|x-y|^{q} \mathrm{e}^{-k(d(x)+d(y))}, \quad \psi(x, y)=u(x)-w(y)-\phi(x, y) .
$$

Let $\left(x_{j}, y_{j}\right) \in \bar{\Omega} \times \bar{\Omega}$ be a maximum point of $\psi$, then $m=\psi(\bar{x}, \bar{x}) \leq u\left(x_{j}\right)-w\left(y_{j}\right)-\phi\left(x_{j}, y_{j}\right)$, from which

$$
\frac{j}{q}\left|x_{j}-y_{j}\right|^{q} \leq\left(u\left(x_{j}\right)-w\left(y_{j}\right)-m\right) \mathrm{e}^{k\left(d\left(x_{j}\right)+d\left(y_{j}\right)\right)} \leq C,
$$

where $C$ is independent of $j$. The last relation implies that, up to subsequence, $x_{j}$ and $y_{j}$ converge to some $\bar{z} \in \bar{\Omega}$ as $j \rightarrow+\infty$. Classical arguments show that

$$
\lim _{j \rightarrow+\infty} \frac{j}{q}\left|x_{j}-y_{j}\right|^{q}=0, \quad \lim _{j \rightarrow+\infty} u\left(x_{j}\right)=u(\bar{z}), \quad \lim _{j \rightarrow+\infty} w\left(y_{j}\right)=w(\bar{z}),
$$

and

$$
u(\bar{z})-w(\bar{z})=m .
$$

Claim 4.12. For $j$ large enough, there exist $x_{j}$ and $y_{j}$ such that $\left(x_{j}, y_{j}\right)$ is a maximum point of $\psi$ and $x_{j} \neq y_{j}$.

Indeed if $x_{j}=y_{j}$ we have

$$
\psi\left(x_{j}, x\right)=u\left(x_{j}\right)-w(x)-\frac{j}{q}\left|x-x_{j}\right|^{q} \mathrm{e}^{-k\left(d\left(x_{j}\right)+d(x)\right)} \leq \psi\left(x_{j}, x_{j}\right)=u\left(x_{j}\right)-w\left(x_{j}\right),
$$

and

$$
\psi\left(x, x_{j}\right)=u(x)-w\left(x_{j}\right)-\frac{j}{q}\left|x-x_{j}\right|^{q} \mathrm{e}^{-k\left(d(x)+d\left(x_{j}\right)\right)} \leq \psi\left(x_{j}, x_{j}\right)=u\left(x_{j}\right)-w\left(x_{j}\right) .
$$

Then $x_{j}$ is a minimum point for

$$
W(x):=w(x)+\frac{j}{q} \mathrm{e}^{-k d\left(x_{j}\right)}\left|x-x_{j}\right|^{q} \mathrm{e}^{-k d(x)},
$$

and a maximum point for

$$
U(x):=u(x)-\frac{j}{q} \mathrm{e}^{-k d\left(x_{j}\right)}\left|x-x_{j}\right|^{q} \mathrm{e}^{-k d(x)} .
$$

We first exclude that $x_{j}$ is both a strict local minimum and a strict local maximum. Indeed in that case, if $u$ and $w$ are not locally constant around $x_{j}$, by Lemma 4.10

$$
\left(c\left(x_{j}\right)+\tau\right) w\left(x_{j}\right) \leq\left(c\left(x_{j}\right)+\lambda\right) u\left(x_{j}\right) .
$$


The same result holds if $u$ or $w$ are locally constant by definition of sub- and supersolution. The last inequality leads to a contradiction, as we will see at the end of the proof. Hence $x_{j}$ cannot be both a strict local minimum and a strict local maximum. In the first case there exist $\delta>0$ and $R>\delta$ such that

$$
\begin{aligned}
w\left(x_{j}\right) & =\min _{\substack{\delta \leq\left|x-x_{j}\right| \leq R \\
x \in \bar{\Omega}}}\left(w(x)+\frac{j}{q}\left|x-x_{j}\right|^{q} \mathrm{e}^{-k\left(d\left(x_{j}\right)+d(x)\right)}\right) \\
& =w\left(y_{j}\right)+\frac{j}{q}\left|y_{j}-x_{j}\right|^{q} \mathrm{e}^{-k\left(d\left(x_{j}\right)+d\left(y_{j}\right)\right)},
\end{aligned}
$$

for some $y_{j} \neq x_{j}$, so that $\left(x_{j}, y_{j}\right)$ is still a maximum point for $\psi$. In the other case, similarly, one can replace $x_{j}$ by a point $y_{j} \neq x_{j}$ such that $\left(y_{j}, x_{j}\right)$ is a maximum for $\psi$. This concludes the Claim 4.12.

Now computing the derivatives of $\phi$ we get

$$
D_{x} \phi(x, y)=j|x-y|^{q-2} \mathrm{e}^{-k(d(x)+d(y))}(x-y)-k \frac{j}{q}|x-y|^{q} \mathrm{e}^{-k(d(x)+d(y))} D d(x),
$$

and

$$
D_{y} \phi(x, y)=-j|x-y|^{q-2} \mathrm{e}^{-k(d(x)+d(y))}(x-y)-k \frac{j}{q}|x-y|^{q} \mathrm{e}^{-k(d(x)+d(y))} D d(y) .
$$

Denote $p_{j}:=D_{x} \phi\left(x_{j}, y_{j}\right)$ and $r_{j}:=-D_{y} \phi\left(x_{j}, y_{j}\right)$. Since $x_{j} \neq y_{j}, p_{j}$ and $r_{j}$ are different from 0 for $j$ large enough. Indeed

$$
0<\frac{j}{2}\left|x_{j}-y_{j}\right|^{q-1} \mathrm{e}^{-2 k d_{0}} \leq\left|p_{j}\right|,\left|r_{j}\right| \leq 2 j\left|x_{j}-y_{j}\right|^{q-1}
$$

for large $j$, where $d_{0}=\max _{\bar{\Omega}} d(x)$. Using (2.2), if $x_{j} \in \partial \Omega$ then

$$
\left\langle p_{j}, \vec{n}\left(x_{j}\right)\right\rangle \geq j\left|x_{j}-y_{j}\right|^{q} \mathrm{e}^{-k d\left(y_{j}\right)}\left(-\frac{1}{2 r}+\frac{k}{q}\right)>0,
$$

and if $y_{j} \in \partial \Omega$ then

$$
\left\langle r_{j}, \vec{n}\left(y_{j}\right)\right\rangle \leq j\left|x_{j}-y_{j}\right|^{q} \mathrm{e}^{-k d\left(x_{j}\right)}\left(\frac{1}{2 r}-\frac{k}{q}\right)<0,
$$

since $k>q /(2 r)$ and $x_{j} \neq y_{j}$. In view of definition of sub- and supersolution we conclude that

$$
\begin{aligned}
& \operatorname{tr}\left(\sigma\left(p_{j}\right) X\right)+b\left(x_{j}\right) \cdot p_{j}+\left(c\left(x_{j}\right)+\lambda\right) u\left(x_{j}\right) \geq 0 \quad \text { if }\left(p_{j}, X\right) \in \bar{J}^{2,+} u\left(x_{j}\right), \\
& \operatorname{tr}\left(\sigma\left(r_{j}\right) Y\right)+b\left(y_{j}\right) \cdot r_{j}+\left(c\left(y_{j}\right)+\tau\right) w\left(y_{j}\right) \leq 0 \quad \text { if }\left(r_{j}, Y\right) \in \bar{J}^{2,-} w\left(y_{j}\right) .
\end{aligned}
$$

Applying Theorem 3.2 of [6] for any $\epsilon>0$ there exist $X_{j}, Y_{j} \in S(N)$ such that $\left(p_{j}, X_{j}\right) \in \bar{J}^{2,+} u\left(x_{j}\right)$, $\left(r_{j}, Y_{j}\right) \in \bar{J}^{2,-} w\left(y_{j}\right)$ and

$$
-\left(\frac{1}{\epsilon}+\left\|D^{2} \phi\left(x_{j}, y_{j}\right)\right\|\right)\left(\begin{array}{cc}
I & 0 \\
0 & I
\end{array}\right) \leq\left(\begin{array}{cc}
X_{j} & 0 \\
0 & -Y_{j}
\end{array}\right) \leq D^{2} \phi\left(x_{j}, y_{j}\right)+\epsilon\left(D^{2} \phi\left(x_{j}, y_{j}\right)\right)^{2} .
$$

Claim 4.13. $X_{j}$ and $Y_{j}$ satisfy

$$
\left(\begin{array}{cc}
X_{j}-\widetilde{X_{j}} & 0 \\
0 & -Y_{j}+\widetilde{Y_{j}}
\end{array}\right) \leq \zeta_{j}\left(\begin{array}{cc}
I & -I \\
-I & I
\end{array}\right),
$$

where $\zeta_{j}=C j\left|x_{j}-y_{j}\right|^{q-2}$, for some positive constant $C$ independent of $j$ and some matrices $\widetilde{X_{j}}, \widetilde{Y_{j}}=O\left(j \mid x_{j}-\right.$ $\left.\left.y_{j}\right|^{q}\right)$. 
To prove the claim we need to estimate $D^{2} \phi\left(x_{j}, y_{j}\right)$ :

$$
\begin{aligned}
D^{2} \phi\left(x_{j}, y_{j}\right)= & \frac{j}{q}\left|x_{j}-y_{j}\right|^{q} D^{2}\left(\mathrm{e}^{-k\left(d\left(x_{j}\right)+d\left(y_{j}\right)\right)}\right)+D\left(\mathrm{e}^{-k\left(d\left(x_{j}\right)+d\left(y_{j}\right)\right)}\right) \otimes \frac{j}{q} D\left(\left|x_{j}-y_{j}\right|^{q}\right) \\
& +\frac{j}{q} D\left(\left|x_{j}-y_{j}\right|^{q}\right) \otimes D\left(\mathrm{e}^{-k\left(d\left(x_{j}\right)+d\left(y_{j}\right)\right)}\right)+\mathrm{e}^{-k\left(d\left(x_{j}\right)+d\left(y_{j}\right)\right)} \frac{j}{q} D^{2}\left(\left|x_{j}-y_{j}\right|^{q}\right) .
\end{aligned}
$$

We denote

$$
\begin{aligned}
& A_{1}:=\frac{j}{q}\left|x_{j}-y_{j}\right|^{q} D^{2}\left(\mathrm{e}^{-k\left(d\left(x_{j}\right)+d\left(y_{j}\right)\right)}\right), \\
& A_{2}:=D \mathrm{e}^{-k\left(d\left(x_{j}\right)+d\left(y_{j}\right)\right)} \otimes \frac{j}{q} D\left(\left|x_{j}-y_{j}\right|^{q}\right)+\frac{j}{q} D\left(\left|x_{j}-y_{j}\right|^{q}\right) \otimes D\left(\mathrm{e}^{-k\left(d\left(x_{j}\right)+d\left(y_{j}\right)\right)}\right), \\
& A_{3}:=\mathrm{e}^{-k\left(d\left(x_{j}\right)+d\left(y_{j}\right)\right)} \frac{j}{q} D^{2}\left(\left|x_{j}-y_{j}\right|^{q}\right) .
\end{aligned}
$$

For $A_{1}$ and $A_{3}$ we have

$$
\begin{gathered}
A_{1} \leq C j\left|x_{j}-y_{j}\right|^{q}\left(\begin{array}{cc}
I & 0 \\
0 & I
\end{array}\right), \\
A_{3} \leq(q-1) j\left|x_{j}-y_{j}\right|^{q-2}\left(\begin{array}{cc}
I & -I \\
-I & I
\end{array}\right) .
\end{gathered}
$$

Here and henceforth, as usual, the letter $C$ denotes various constants independent of $j$. Now we consider the quantity $\left\langle A_{2}(\xi, \eta),(\xi, \eta)\right\rangle$ for $\xi, \eta \in \mathbb{R}^{N}$. We have

$$
\begin{aligned}
\left\langle A_{2}(\xi, \eta),(\xi, \eta)\right\rangle= & 2 k j\left|x_{j}-y_{j}\right|^{q-2} \mathrm{e}^{-k\left(d\left(x_{j}\right)+d\left(y_{j}\right)\right)}\left[\left\langle D d\left(x_{j}\right) \otimes\left(x_{j}-y_{j}\right)(\eta-\xi), \xi\right\rangle\right. \\
& \left.+\left\langle D d\left(y_{j}\right) \otimes\left(x_{j}-y_{j}\right)(\eta-\xi), \eta\right\rangle\right] \\
\leq & C j\left|x_{j}-y_{j}\right|^{q-1}|\xi-\eta|(|\xi|+|\eta|) \\
\leq & C j\left|x_{j}-y_{j}\right|^{q-1}\left(\frac{|\xi-\eta|^{2}}{\left|x_{j}-y_{j}\right|}+\frac{(|\xi|+|\eta|)^{2}}{4}\left|x_{j}-y_{j}\right|\right) \\
\leq & C\left[j\left|x_{j}-y_{j}\right|^{q-2}|\xi-\eta|^{2}+j\left|x_{j}-y_{j}\right|^{q}\left(|\xi|^{2}+|\eta|^{2}\right)\right] .
\end{aligned}
$$

The last inequality can be rewritten equivalently in this way

$$
A_{2} \leq C j\left|x_{j}-y_{j}\right|^{q-2}\left(\begin{array}{cc}
I & -I \\
-I & I
\end{array}\right)+C j\left|x_{j}-y_{j}\right|^{q}\left(\begin{array}{cc}
I & 0 \\
0 & I
\end{array}\right) .
$$

Finally if we choose

$$
\epsilon=\frac{1}{j\left|x_{j}-y_{j}\right|^{q-2}},
$$

we get the same estimates for the matrix $\epsilon\left(D^{2} \phi\left(x_{j}, y_{j}\right)\right)^{2}$. In conclusion we have

$$
D^{2} \phi\left(x_{j}, y_{j}\right)+\epsilon\left(D^{2} \phi\left(x_{j}, y_{j}\right)\right)^{2} \leq C j\left|x_{j}-y_{j}\right|^{q-2}\left(\begin{array}{cc}
I & -I \\
-I & I
\end{array}\right)+C j\left|x_{j}-y_{j}\right|^{q}\left(\begin{array}{cc}
I & 0 \\
0 & I
\end{array}\right),
$$

and (4.8) implies (4.9). The Claim 4.13 is proved.

Now, multiplying the inequality (4.9) on the left for the non-negative symmetric matrix

$$
\left(\begin{array}{cc}
\sigma\left(p_{j}\right) \sigma\left(p_{j}\right) & \sigma\left(p_{j}\right) \sigma\left(r_{j}\right) \\
\sigma\left(r_{j}\right) \sigma\left(p_{j}\right) & \sigma\left(r_{j}\right) \sigma\left(r_{j}\right)
\end{array}\right)=\left(\begin{array}{cc}
\sigma\left(p_{j}\right) & \sigma\left(p_{j}\right) \sigma\left(r_{j}\right) \\
\sigma\left(r_{j}\right) \sigma\left(p_{j}\right) & \sigma\left(r_{j}\right)
\end{array}\right)
$$


taking traces and using (2.1) and (4.7), we get

$$
\begin{aligned}
\operatorname{tr}\left(\sigma\left(p_{j}\right)\left(X_{j}-\widetilde{X}_{j}\right)\right)-\operatorname{tr}\left(\sigma\left(r_{j}\right)\left(Y_{j}-\widetilde{Y_{j}}\right)\right) & \leq \zeta_{j} \operatorname{tr}\left[\left(\sigma\left(p_{j}\right)-\sigma\left(r_{j}\right)\right)^{2}\right] \leq \frac{8 \zeta_{j}}{\left|p_{j}\right|^{2}}\left|p_{j}-r_{j}\right|^{2} \\
& \leq C \frac{j\left|x_{j}-y_{j}\right|^{q-2} j^{2}\left|x_{j}-y_{j}\right|^{2 q}}{j^{2}\left|x_{j}-y_{j}\right|^{2(q-1)}} \\
& =C j\left|x_{j}-y_{j}\right|^{q} .
\end{aligned}
$$

Now using that $u$ and $w$ are respectively sub- and supersolution we compute

$$
\begin{aligned}
-\left(\lambda+c\left(x_{j}\right)\right) u\left(x_{j}\right) & \leq \operatorname{tr}\left(\sigma\left(p_{j}\right) X_{j}\right)+b\left(x_{j}\right) \cdot p_{j} \\
& \leq \operatorname{tr}\left(\sigma\left(p_{j}\right)\left(X_{j}-\widetilde{X}_{j}\right)\right)+b\left(x_{j}\right) \cdot p_{j}+O\left(j\left|x_{j}-y_{j}\right|^{q}\right) \\
& \leq \operatorname{tr}\left(\sigma\left(r_{j}\right)\left(Y_{j}-\widetilde{Y}_{j}\right)\right)+b\left(x_{j}\right) \cdot p_{j}+O\left(j\left|x_{j}-y_{j}\right|^{q}\right) \\
& \leq-\left(\tau+c\left(y_{j}\right)\right) w\left(y_{j}\right)+b\left(x_{j}\right) \cdot p_{j}-b\left(y_{j}\right) \cdot r_{j}+O\left(j\left|x_{j}-y_{j}\right|^{q}\right) .
\end{aligned}
$$

The quantity $b\left(x_{j}\right) \cdot p_{j}-b\left(y_{j}\right) \cdot r_{j}$ goes to 0 as $j \rightarrow+\infty$. Indeed, since $m>0$ and $w$ is positive and bounded, the estimate (3.1) of Lemma 3.2 holds for $u$ and $w$; using it in (4.6) and dividing by $\left|x_{j}-y_{j}\right| \neq 0$ we obtain

$$
\frac{j}{q}\left|x_{j}-y_{j}\right|^{q-1} \leq C_{0} \mathrm{e}^{k\left(d\left(x_{j}\right)+d\left(y_{j}\right)\right)} \leq C .
$$

Then by (4.7) we conclude that the sequences $\left\{p_{j}\right\}$ and $\left\{r_{j}\right\}$ are bounded, so that, since in addition $\left|p_{j}-r_{j}\right| \leq$ $C j\left|x_{j}-y_{j}\right|^{q} \rightarrow 0$ as $j \rightarrow+\infty$, up to subsequence $p_{j}, r_{j} \rightarrow p_{0}$ as $j \rightarrow+\infty$.

Hence, sending $j \rightarrow+\infty$ we obtain

$$
-(\lambda+c(\bar{z})) u(\bar{z}) \leq-(\tau+c(\bar{z})) w(\bar{z}) .
$$

If $\tau+c(\bar{z})>0$, using (4.5) we get

$$
-(\lambda+c(\bar{z})) u(\bar{z}) \leq-(\tau+c(\bar{z})) \frac{\gamma}{\gamma^{\prime}} u(\bar{z})
$$

and taking $\gamma$ sufficiently close to $\gamma^{\prime}$ in order that $\frac{\tau \frac{\gamma}{\gamma^{\prime}}-\lambda}{1-\frac{\gamma}{\gamma^{\prime}}}>|c|_{\infty}$, we obtain a contradiction. Finally if $\tau+c(\bar{z}) \leq 0$ we have

$$
-(\lambda+c(\bar{z})) u(\bar{z}) \leq-(\tau+c(\bar{z})) w(\bar{z}) \leq-(\tau+c(\bar{z})) u(\bar{z}),
$$

once more a contradiction since $\lambda<\tau$.

Proof of Lemma 4.10. Without loss of generality we can assume that $\bar{x}=0$.

Since the minimum is strict there exists a small $\delta>0$ such that

$$
v(0)<v(x)+C|x|^{q} \mathrm{e}^{-k d(x)} \quad \text { for any } x \in \bar{\Omega}, 0<|x| \leq \delta .
$$

Since $v$ is not locally constant and $q>1$ for any $n>\delta^{-1}$ there exists $\left(t_{n}, z_{n}\right) \in B\left(0, \frac{1}{n}\right)^{2} \cap \bar{\Omega}^{2}$ such that

$$
v\left(t_{n}\right)>v\left(z_{n}\right)+C\left|z_{n}-t_{n}\right|^{q} \mathrm{e}^{-k d\left(z_{n}\right)} .
$$

Consequently, for $n>\delta^{-1}$ the minimum of the function $v(x)+C\left|x-t_{n}\right|^{q} \mathrm{e}^{-k d(x)}$ in $\bar{B}(0, \delta) \cap \bar{\Omega}$ is not achieved on $t_{n}$. Indeed

$$
\min _{|x| \leq \delta, x \in \bar{\Omega}}\left(v(x)+C\left|x-t_{n}\right|^{q} \mathrm{e}^{-k d(x)}\right) \leq v\left(z_{n}\right)+C\left|z_{n}-t_{n}\right|^{q} \mathrm{e}^{-k d\left(z_{n}\right)}<v\left(t_{n}\right) .
$$


Let $y_{n} \neq t_{n}$ be some point in $\bar{B}(0, \delta) \cap \bar{\Omega}$ on which the minimum is achieved. Passing to the limit as $n$ goes to infinity, $t_{n}$ goes to 0 and, up to subsequence, $y_{n}$ converges to some $y \in \bar{B}(0, \delta) \cap \bar{\Omega}$. By the lower semicontinuity of $v$ and the fact that 0 is a local minimum of $v(x)+C|x|^{q} \mathrm{e}^{-k d(x)}$ we have

$$
v(0) \leq v(y)+C|y|^{q} \mathrm{e}^{-k d(y)} \leq \liminf _{n \rightarrow+\infty}\left(v\left(y_{n}\right)+C\left|y_{n}\right|^{q} \mathrm{e}^{-k d\left(y_{n}\right)}\right),
$$

and using that $v(0)+C\left|t_{n}\right|^{q} \mathrm{e}^{-k d(0)} \geq v\left(y_{n}\right)+C\left|y_{n}-t_{n}\right|^{q} \mathrm{e}^{-k d\left(y_{n}\right)}$, one has

$$
v(0) \geq \limsup _{n \rightarrow+\infty}\left(v\left(y_{n}\right)+C\left|y_{n}\right|^{q} \mathrm{e}^{-k d\left(y_{n}\right)}\right) .
$$

Then

$$
v(0)=v(y)+C|y|^{q} \mathrm{e}^{-k d(y)}=\lim _{n \rightarrow+\infty}\left(v\left(y_{n}\right)+C\left|y_{n}\right|^{q} \mathrm{e}^{-k d\left(y_{n}\right)}\right) .
$$

Since 0 is a strict local minimum of $v(x)+C|x|^{q} \mathrm{e}^{-k d(x)}$, the last equalities imply that $y=0$ and $v\left(y_{n}\right)$ goes to $v(0)$ as $n \rightarrow+\infty$. Then for large $n, y_{n}$ is an interior point of $B(0, \delta)$ so that the function

$$
\varphi(x)=v\left(y_{n}\right)+C\left|y_{n}-t_{n}\right|^{q} \mathrm{e}^{-k d\left(y_{n}\right)}-C\left|x-t_{n}\right|^{q} \mathrm{e}^{-k d(x)}
$$

is a test function for $v$ at $y_{n}$. Moreover, the gradient of $\varphi$

$$
D \varphi(x)=-C q\left|x-t_{n}\right|^{q-2} \mathrm{e}^{-k d(x)}\left(x-t_{n}\right)+k C\left|x-t_{n}\right|^{q} \mathrm{e}^{-k d(x)} D d(x)
$$

is different from 0 at $x=y_{n}$ for small $\delta$, indeed

$$
\left|D \varphi\left(y_{n}\right)\right| \geq C\left|y_{n}-t_{n}\right|^{q-1} \mathrm{e}^{-k d\left(y_{n}\right)}\left(q-k\left|y_{n}-t_{n}\right|\right) \geq C\left|y_{n}-t_{n}\right|^{q-1} \mathrm{e}^{-k d\left(y_{n}\right)}(q-2 k \delta)>0 .
$$

Using (2.2), if $y_{n} \in \partial \Omega$ we have

$$
\left\langle D \varphi\left(y_{n}\right), \vec{n}\left(y_{n}\right)\right\rangle \leq C\left|y_{n}-t_{n}\right|^{q}\left(\frac{q}{2 r}-k\right)<0,
$$

since $k>q /(2 r)$. Then we conclude that

$$
\operatorname{tr}\left(\sigma\left(D \varphi\left(y_{n}\right)\right) D^{2} \varphi\left(y_{n}\right)\right)+b\left(y_{n}\right) \cdot D \varphi\left(y_{n}\right)-\beta\left(v\left(y_{n}\right)\right) \leq g\left(y_{n}\right) .
$$

Observe that $D^{2} \varphi\left(y_{n}\right)=\left|y_{n}-t_{n}\right|^{q-2} M$, where $M$ is a bounded matrix. Hence, from the last inequality we get

$$
C_{0}\left|y_{n}-t_{n}\right|^{q-2}-\beta\left(v\left(y_{n}\right)\right) \leq g\left(y_{n}\right),
$$

for some constant $C_{0}$. Passing to the limit, since $\beta$ and $g$ are upper semicontinuous we obtain

$$
-\beta(v(0)) \leq g(0),
$$

which is the desired conclusion.

We conclude sketching the proof of Theorem 4.1.

Proof of Theorem 4.1. Suppose by contradiction that $\max _{\bar{\Omega}}(u-v)=m>0$. Since $u \leq v$ on the boundary, the supremum is achieved inside $\Omega$. Let us define for $j \in \mathbb{N}$ and some $q>2$

$$
\psi(x, y)=u(x)-v(y)-\frac{j}{q}|x-y|^{q} .
$$


Suppose that $\left(x_{j}, y_{j}\right)$ is a maximum point for $\psi$ in $\bar{\Omega}^{2}$. Then $\left|x_{j}-y_{j}\right| \rightarrow 0$ as $j \rightarrow+\infty$ and up to subsequence $x_{j}, y_{j} \rightarrow \bar{x}, u\left(x_{j}\right) \rightarrow u(\bar{x}), v\left(y_{j}\right) \rightarrow v(\bar{x})$ and $j\left|x_{j}-y_{j}\right|^{q} \rightarrow 0$ as $j \rightarrow+\infty$. Moreover, $\bar{x}$ is such that $u(\bar{x})-v(\bar{x})=m$ and we can choose $x_{j} \neq y_{j}$. Recalling by Remark 3.3 that the estimate (3.1) holds in $\Omega$, we can proceed as in the proof of Theorem 4.8 to get

This is a contradiction since $c(\bar{x})<0$.

$$
-c(\bar{x}) u(\bar{x}) \leq-c(\bar{x}) v(\bar{x}) .
$$

\subsection{The maximum principle for $c(x)$ changing sign: an example}

In the previous subsections we have proved that $\Delta_{\infty}+b(x) \cdot D+c(x)$ with the Neumann boundary condition satisfies the maximum principle if $c(x) \leq 0$ or without condition on the sign of $c(x)$ provided $\bar{\lambda}>0$. In this subsection we want to show that these two cases do not coincide, i.e., that there exists some $c(x)$ which changes sign in $\Omega$ such that the associated principal eigenvalue $\bar{\lambda}$ is positive. To prove this, by definition of $\bar{\lambda}$, it suffices to find a function $c(x)$ changing sign for which there exists a bounded positive supersolution of

$$
\begin{cases}\Delta_{\infty} v+b(x) \cdot D v+(c(x)+\lambda) v=0 & \text { in } \Omega \\ \frac{\partial v}{\partial \vec{n}}=0 & \text { on } \partial \Omega\end{cases}
$$

for some $\lambda>0$. For simplicity, let us suppose that $b \equiv 0$ and $\Omega$ is the ball of center 0 and radius $R$. We will look for $c$ such that:

$$
\begin{cases}c(x)<0 & \text { if } R-\epsilon<|x| \leq R \\ c(x) \leq-\beta_{1} & \text { if } \rho<|x| \leq R-\epsilon \\ c(x) \leq \beta_{2} & \text { if }|x| \leq \rho\end{cases}
$$

where $0<\rho<R-\epsilon$ and $\epsilon, \beta_{1}, \beta_{2}$ are positive constants. Remark that in the ball of radius $\rho, c(x)$ may assume positive values. Following [16], it is possible to construct a supersolution of (4.10) if $\epsilon$ is small enough and

$$
\beta_{2}<\frac{k^{2} \mathrm{e}^{-k \rho}}{\frac{k}{4}(R-\rho)+\frac{2 k}{\beta_{1}(R-\rho)}+1-\mathrm{e}^{-k \rho}}
$$

for some $k>0$. From the last relation we can see that choosing $k=\frac{1}{\rho}$ the term on the right-hand side goes to $+\infty$ as $\rho \rightarrow 0^{+}$, that is, if the set where $c_{0}(x)$ is positive becomes smaller then the values of $c_{0}(x)$ in this set can be very large. On the contrary, for any value of $k$, if $\rho \rightarrow R^{-}$then $\beta_{2}$ goes to 0 . Finally for any $k$ if $\beta_{1} \rightarrow 0^{+}$, then again $\beta_{2}$ goes to 0 .

\section{Some eXistence Results}

This section is devoted to the problem of the existence of a solution of

$$
\begin{cases}\Delta_{\infty} u+b(x) \cdot D u+(c(x)+\lambda) u=g(x) & \text { in } \Omega \\ \frac{\partial u}{\partial \vec{n}}=0 & \text { on } \partial \Omega .\end{cases}
$$

The first existence result for (5.1) is obtained when $\lambda=0$ and $c<0$, via Perron's method. Then, we will prove the existence of a positive solution of (5.1) when $g$ is non-positive and $\lambda<\bar{\lambda}$ (without condition on the sign of $c$ ). These two results will allow us to prove that the Neumann problem (5.1) is solvable for any right-hand side if $\lambda<\bar{\lambda}$. Finally, we will prove the existence of a positive principal eigenfunction corresponding to $\bar{\lambda}$, that is a solution of (5.1) when $g \equiv 0$ and $\lambda=\bar{\lambda}$. 
Comparison results guarantee for (5.1) the uniqueness of the solution when $c<0$ and when $\lambda<\bar{\lambda}$ and $g<0$ or $g>0$.

Theorem 5.1. Suppose that $c<0$ and $g$ is continuous on $\bar{\Omega}$. If $u \in U S C(\bar{\Omega})$ and $v \in L S C(\bar{\Omega})$ are respectively viscosity sub- and supersolution of

$$
\begin{cases}\Delta_{\infty} u+b(x) \cdot D u+c(x) u=g(x) & \text { in } \Omega \\ \frac{\partial u}{\partial \vec{n}}=0 & \text { on } \partial \Omega\end{cases}
$$

with $u$ and $v$ bounded or $v \geq 0$ and bounded, then $u \leq v$ on $\bar{\Omega}$. Moreover (5.2) has a unique viscosity solution.

Proof. We suppose by contradiction that $\max _{\bar{\Omega}}(u-v)=m>0$. Repeating the proof of Theorem 4.8 taking $v$ as $w$, we arrive to the following inequality

$$
-c(\bar{z}) u(\bar{z}) \leq-c(\bar{z}) v(\bar{z}),
$$

where $\bar{z} \in \bar{\Omega}$ is such that $u(\bar{z})-v(\bar{z})=m>0$. This is a contradiction since $c(\bar{z})<0$.

The existence of a solution follows from Perron's method of Ishii, see e.g. [6], and the comparison result just proved, provided there is a bounded subsolution and a bounded supersolution of (5.2). Since $c$ is negative and continuous on $\bar{\Omega}$, there exists $c_{0}>0$ such that $c(x) \leq-c_{0}$ for every $x \in \bar{\Omega}$. Then

$$
u_{1}:=-\frac{|g|_{\infty}}{c_{0}}, \quad u_{2}:=\frac{|g|_{\infty}}{c_{0}}
$$

are respectively a bounded sub- and supersolution of (5.2).

Define

$$
u(x):=\sup \left\{\varphi(x) \mid u_{1} \leq \varphi \leq u_{2} \text { and } \varphi\right. \text { is a subsolution of (5.2)\}, }
$$

we claim that $u$ is a solution of (5.2). We first show that the upper semicontinuous envelope of $u$ defined as

$$
u^{*}(x):=\lim _{\rho \downarrow 0} \sup \{u(y): y \in \bar{\Omega} \text { and }|y-x| \leq \rho\}
$$

is a subsolution of (5.2). Indeed if $(p, X) \in J^{2,+} u\left(x_{0}\right)$ and $p \neq 0$ then by the standard arguments of the Perron's method it can be proved that $\operatorname{tr}(\sigma(p) X)+b\left(x_{0}\right) \cdot p+c\left(x_{0}\right) u\left(x_{0}\right) \geq g\left(x_{0}\right)$ if $x_{0} \in \Omega$ and $(-\operatorname{tr}(\sigma(p) X)-$ $\left.b\left(x_{0}\right) \cdot p-c\left(x_{0}\right) u\left(x_{0}\right)+g\left(x_{0}\right)\right) \wedge\left\langle p, \vec{n}\left(x_{0}\right)\right\rangle \leq 0$ if $x_{0} \in \partial \Omega$.

Now suppose $u^{*} \equiv k$ in a neighborhood of $x_{0} \in \bar{\Omega}$. If $x_{0} \in \partial \Omega$ clearly $u^{*}$ is subsolution at $x_{0}$. Assume that $x_{0}$ is an interior point of $\Omega$. We may choose a sequence of subsolutions $\left(\varphi_{n}\right)_{n}$ and a sequence of points $\left(x_{n}\right)_{n}$ in $\Omega$ such that $x_{n} \rightarrow x_{0}$ and $\varphi_{n}\left(x_{n}\right) \rightarrow k$. Suppose that $\left|x_{n}-x_{0}\right|<a_{n}$ with $a_{n}$ decreasing to 0 as $n \rightarrow+\infty$. If, up to subsequence, $\varphi_{n}$ is constant in $B\left(x_{0}, a_{n}\right)$ for any $n$, then passing to the limit in the relation $c\left(x_{n}\right) \varphi_{n}\left(x_{n}\right) \geq g\left(x_{n}\right)$ we get $c\left(x_{0}\right) k \geq g\left(x_{0}\right)$ as desired. Otherwise, suppose that for any $n \varphi_{n}$ is not constant in $B\left(x_{0}, a_{n}\right)$. Repeating the argument of Lemma 4.10 we find a sequence $\left\{\left(t_{n}, y_{n}\right)\right\}_{n \in \mathbb{N}} \subset \Omega^{2}$ and a small $\delta>0$ such that $\left|t_{n}-x_{0}\right|<a_{n},\left|y_{n}-x_{0}\right| \leq \delta, t_{n} \neq y_{n}, \varphi_{n}(x)-\left|x-t_{n}\right|^{q} \leq \varphi_{n}\left(y_{n}\right)-\left|y_{n}-t_{n}\right|^{q}$ for any $x \in B\left(x_{0}, \delta\right)$, with $q>2$ and $u^{*} \equiv k$ in $\bar{B}\left(x_{0}, \delta\right)$. Up to subsequence $y_{n} \rightarrow y \in \bar{B}\left(x_{0}, \delta\right)$ as $n \rightarrow+\infty$. We have

$$
\begin{aligned}
k & =\lim _{n \rightarrow+\infty}\left(\varphi_{n}\left(x_{n}\right)-\left|x_{n}-t_{n}\right|^{q}\right) \leq \liminf _{n \rightarrow+\infty}\left(\varphi_{n}\left(y_{n}\right)-\left|y_{n}-t_{n}\right|^{q}\right) \\
& \leq \limsup _{n \rightarrow+\infty}\left(\varphi_{n}\left(y_{n}\right)-\left|y_{n}-t_{n}\right|^{q}\right) \leq k-\left|y-x_{0}\right|^{q} .
\end{aligned}
$$

The last inequalities imply that $y=x_{0}$ and $\varphi_{n}\left(y_{n}\right) \rightarrow k$. Then, for large $n, y_{n}$ is an interior point of $B\left(x_{0}, \delta\right)$ and $\phi_{n}(x):=\varphi_{n}\left(y_{n}\right)-\left|y_{n}-t_{n}\right|^{q}+\left|x-t_{n}\right|^{q}$ is a test function for $\varphi_{n}$ at $y_{n}$. Passing to the limit as $n \rightarrow+\infty$ 
in the relation $\Delta_{\infty} \phi_{n}\left(y_{n}\right)+b\left(y_{n}\right) \cdot D \phi_{n}\left(y_{n}\right)+c\left(y_{n}\right) \varphi_{n}\left(y_{n}\right) \geq g\left(y_{n}\right)$, we get again $c\left(x_{0}\right) k \geq g\left(x_{0}\right)$. In conclusion $u^{*}$ is a subsolution of (5.2). Since $u^{*} \leq u_{2}$, it follows from the definition of $u$ that $u=u^{*}$.

Finally the lower semicontinuous envelope of $u$ defined as

$$
u_{*}(x):=\lim _{\rho \downarrow 0} \inf \{u(y): y \in \bar{\Omega} \text { and }|y-x| \leq \rho\}
$$

is a supersolution. Indeed, if it is not, the Perron's method provides a viscosity subsolution of (5.2) greater than $u$, contradicting the definition of $u$. If $u_{*} \equiv k$ in a neighborhood of $x_{0} \in \Omega$ and $c\left(x_{0}\right) k>g\left(x_{0}\right)$ then for small $\delta$ and $\rho$, the subsolution is

$$
u_{\delta, \rho}(x):= \begin{cases}\max \left\{u(x), k+\frac{\delta \rho^{2}}{8}-\delta\left|x-x_{0}\right|^{2}\right\} & \text { if }\left|x-x_{0}\right|<\rho, \\ u(x) & \text { otherwise. }\end{cases}
$$

Hence $u_{*}$ is a supersolution of (5.2) and then, by comparison, $u^{*}=u \leq u_{*}$, showing that $u$ is continuous and is a solution.

The uniqueness of the solution is an immediate consequence of the comparison principle just proved.

Theorem 5.2. Suppose $g \in L S C(\bar{\Omega}), h \in U S C(\bar{\Omega}), h \leq 0, h \leq g$ and $g(x)>0$ if $h(x)=0$. Let $u \in U S C(\bar{\Omega})$ be a viscosity subsolution of (5.1) and $v \in L S C(\bar{\Omega})$ be a bounded positive viscosity supersolution of (5.1) with $g$ replaced by $h$. Then $u \leq v$ on $\bar{\Omega}$.

Remark 5.3. The existence of a such $v$ implies $\lambda \leq \bar{\lambda}$.

Proof. It suffices to prove the theorem for $h<g$. Indeed, for $l>1$ the function $l v$ is a supersolution of (5.1) with right-hand side $l h(x)$ and by the assumptions on $h$ and $g, l h<g$. If $u \leq l v$ for any $l>1$, passing to the limit as $l \rightarrow 1^{+}$, one obtains $u \leq v$ as desired.

Hence we can assume $h<g$. By upper semicontinuity $\max _{\bar{\Omega}}(h-g)=-M<0$. Suppose by contradiction that $u>v$ somewhere in $\bar{\Omega}$. Then there exists $\bar{y} \in \bar{\Omega}$ such that

$$
\gamma^{\prime}:=\frac{u(\bar{y})}{v(\bar{y})}=\max _{x \in \bar{\Omega}} \frac{u(x)}{v(x)}>1 .
$$

Define $w=\gamma v$ for some $1 \leq \gamma<\gamma^{\prime}$. Since $h \leq 0$ and $\gamma \geq 1, \gamma h \leq h$ and then $w$ is still a supersolution of (5.1) with right-hand side $h$. The supremum of $u-w$ is strictly positive then, by upper semicontinuity, there exists $\bar{x} \in \bar{\Omega}$ such that $u(\bar{x})-w(\bar{x})=\max _{\bar{\Omega}}(u-w)>0$. We have $u(\bar{x})>w(\bar{x})$ and $w(\bar{x}) \geq \frac{\gamma}{\gamma^{\prime}} u(\bar{x})$. Repeating the proof of Theorem 4.8, we get

$$
g(\bar{z})-(\lambda+c(\bar{z})) u(\bar{z}) \leq h(\bar{z})-(\lambda+c(\bar{z})) w(\bar{z}),
$$

where $\bar{z}$ is some point in $\bar{\Omega}$ where the maximum of $u-w$ is attained. If $\lambda+c(\bar{z}) \leq 0$, then

$$
-(\lambda+c(\bar{z})) u(\bar{z}) \leq h(\bar{z})-g(\bar{z})-(\lambda+c(\bar{z})) w(\bar{z})<-(\lambda+c(\bar{z})) u(\bar{z}),
$$

which is a contradiction. If $\lambda+c(\bar{z})>0$, then

$$
-(\lambda+c(\bar{z})) u(\bar{z}) \leq h(\bar{z})-g(\bar{z})-(\lambda+c(\bar{z})) \frac{\gamma}{\gamma^{\prime}} u(\bar{z}) .
$$

If we choose $\gamma$ sufficiently close to $\gamma^{\prime}$ in order that

$$
|\lambda+c|_{\infty}\left(\frac{\gamma}{\gamma^{\prime}}-1\right) \max _{\bar{\Omega}} u \geq-\frac{M}{2},
$$

we get once more a contradiction. 
Theorem 5.4. Suppose that $\lambda<\bar{\lambda}, g \leq 0, g \not \equiv 0$ and $g$ is continuous on $\bar{\Omega}$, then there exists a positive viscosity solution of (5.1). If $g<0$, the solution is unique.

Proof. We follow the proof of Theorem 7 of [4].

If $\lambda<-|c|_{\infty}$ then the existence of the solution is guaranteed by Theorem 5.1. Let us suppose $\lambda \geq-|c|_{\infty}$ and define by induction the sequence $\left(u_{n}\right)_{n}$ by $u_{1}=0$ and $u_{n+1}$ as the solution of

$$
\begin{cases}\Delta_{\infty} u_{n+1}+b(x) \cdot D u_{n+1}+\left(c(x)-|c|_{\infty}-1\right) u_{n+1}=g-\left(\lambda+|c|_{\infty}+1\right) u_{n} & \text { in } \Omega \\ \frac{\partial u_{n+1}}{\partial \vec{n}}=0 & \text { on } \partial \Omega\end{cases}
$$

which exists by Theorem 5.1. By the comparison principle, since $g \leq 0$ and $g \not \equiv 0$ the sequence is positive and increasing.

We claim that $\left(u_{n}\right)_{n}$ is also bounded. Suppose that it is not, then dividing by $\left|u_{n+1}\right|_{\infty}$ and defining $v_{n}:=$ $\frac{u_{n}}{\left|u_{n}\right|_{\infty}}$ one gets that $v_{n+1}$ is a solution of

$$
\begin{cases}\Delta_{\infty} v_{n+1}+b(x) \cdot D v_{n+1}+\left(c(x)-|c|_{\infty}-1\right) v_{n+1} & \\ =\frac{g}{\left.u_{n+1}\right|_{\infty}}-\left(\lambda+|c|_{\infty}+1\right) \frac{u_{n}}{\left|u_{n+1}\right|_{\infty}} & \text { in } \Omega \\ \frac{\partial v_{n+1}}{\partial \vec{n}}=0 & \text { on } \partial \Omega .\end{cases}
$$

By Corollary 3.4, $\left(v_{n}\right)_{n}$ converges to a positive function $v$ with $|v|_{\infty}=1$, which satisfies

$$
\begin{cases}\Delta_{\infty} v+b(x) \cdot D v+(c(x)+\lambda) v & \\ =\left(\lambda+|c|_{\infty}+1\right)(1-k) v \geq 0 & \text { in } \Omega \\ \frac{\partial v_{n+1}}{\partial \vec{n}}=0 & \text { on } \partial \Omega\end{cases}
$$

where $k:=\lim _{n \rightarrow+\infty} \frac{\left|u_{n}\right|_{\infty}}{\left|u_{n+1}\right|_{\infty}} \leq 1$. This contradicts the maximum principle, Theorem 4.8.

Then $\left(u_{n}\right)_{n}$ is bounded and letting $n$ go to infinity, by the compactness result, the sequence converges to a function $u$ which is a solution. Moreover, the solution is positive on $\bar{\Omega}$ by the strong minimum principle, Proposition 4.2.

If $g<0$, the uniqueness of the solution follows from Theorem 5.2.

Remark 5.5. Clearly, since the operator $\Delta_{\infty}$ is odd, by Theorem 5.4, there exists a negative solution of (5.1) for $\lambda<\underline{\lambda}$ and $g \geq 0, g \not \equiv 0$, which is unique if $g>0$.

Theorem 5.6. Suppose that $\lambda<\bar{\lambda}$ and $g$ is continuous on $\bar{\Omega}$, then there exists a viscosity solution of (5.1).

Proof. If $g \equiv 0$, by the maximum principle the only solution is $u \equiv 0$. Let us suppose $g \not \equiv 0$. Since $\lambda<\bar{\lambda}$ by Theorem 5.4 there exist $v_{0}$ positive viscosity solution of (5.1) with right-hand side $-|g|_{\infty}$ and $u_{0}$ negative viscosity solution of (5.1) with right-hand side $|g|_{\infty}$.

Let us suppose $\lambda+|c|_{\infty} \geq 0$. Let $\left(u_{n}\right)_{n}$ be the sequence defined in the proof of Theorem 5.4 with $u_{1}=u_{0}$, then by comparison Theorem 5.1 we have $u_{0}=u_{1} \leq u_{2} \leq \ldots \leq v_{0}$. Hence, by the compactness Corollary 3.4 the sequence converges to a continuous function which is the desired solution.

Theorem 5.7 (existence of principal eigenfunctions). There exists $\phi>0$ on $\bar{\Omega}$ viscosity solution of

$$
\begin{cases}\Delta_{\infty} \phi+b(x) \cdot D \phi+(c(x)+\bar{\lambda}) \phi=0 & \text { in } \Omega \\ \frac{\partial \phi}{\partial \vec{n}}=0 & \text { on } \partial \Omega .\end{cases}
$$

Moreover $\phi$ is Lipschitz continuous on $\bar{\Omega}$. 
Proof. Let $\lambda_{n}$ be an increasing sequence which converges to $\bar{\lambda}$. Let $u_{n}$ be the positive solution of (5.1) with $\lambda=\lambda_{n}$ and $g \equiv-1$. By Theorem 5.4 the sequence $\left(u_{n}\right)_{n}$ is well defined. Following the argument of the proof of Theorem 8 of [4], it can proved that it is unbounded, otherwise one would contradict the definition of $\bar{\lambda}$. Then, up to subsequence $\left|u_{n}\right|_{\infty} \rightarrow+\infty$ as $n \rightarrow+\infty$ and defining $v_{n}:=\frac{u_{n}}{\left|u_{n}\right|_{\infty}}$ one gets that $v_{n}$ satisfies (5.1) with $\lambda=\lambda_{n}$ and $g \equiv-\frac{1}{\left|u_{n}\right|_{\infty}}$. Then by Corollary 3.4, we can extract a subsequence converging to a positive function $\phi$ with $|\phi|_{\infty}=1$ which is the desired solution. By Theorem 3.1 the solution is also Lipschitz continuous on $\bar{\Omega}$.

\section{A DECAy EStimate FOR SOLUtions of THE EVOLUtion PROBLEM}

In this section we want to study the asymptotic behavior as $t \rightarrow+\infty$ of the solution $h(t, x)$ of the evolution problem

$$
\begin{cases}h_{t}=\Delta_{\infty} h+c(x) h & \text { in }(0,+\infty) \times \Omega \\ \frac{\partial h}{\partial \vec{n}}=0 & \text { on }[0,+\infty) \times \partial \Omega \\ h(0, x)=h_{0}(x) & \text { for } x \in \Omega\end{cases}
$$

where $h_{0}$ is a continuous function on $\bar{\Omega}$. As in $[11,12]$ we use the semicontinuous extensions of the function $(p, X) \rightarrow \operatorname{tr}(\sigma(p) X)$ to define the viscosity solutions of (6.1). For $X \in S(N)$, let us denote its smaller and larger eigenvalue respectively by $m(X)$ and $M(X)$, that is

$$
\begin{aligned}
& m(X):=\min _{|\xi|=1}\langle X \xi, \xi\rangle, \\
& M(X):=\max _{|\xi|=1}\langle X \xi, \xi\rangle .
\end{aligned}
$$

Definition 6.1. Any function $u \in U S C([0,+\infty) \times \bar{\Omega})$ (resp., $u \in L S C([0,+\infty) \times \bar{\Omega})$ ) is called viscosity subsolution (resp., supersolution) of (6.1) if for any $x \in \bar{\Omega}, u(0, x) \leq h_{0}(x)$ (resp., $u(0, x) \geq h_{0}(x)$ ) and if the following conditions hold:

(i) For every $\left(t_{0}, x_{0}\right) \in(0,+\infty) \times \Omega$, for all $\varphi \in C^{2}([0,+\infty) \times \bar{\Omega})$, such that $u-\varphi$ has a local maximum (resp., minimum) at $\left(t_{0}, x_{0}\right)$, one has

$$
\begin{cases}\varphi_{t}\left(t_{0}, x_{0}\right) \leq \Delta_{\infty} \varphi\left(t_{0}, x_{0}\right)+c\left(x_{0}\right) u\left(t_{0}, x_{0}\right)(\text { resp. }, \geq) & \text { if } D \varphi\left(t_{0}, x_{0}\right) \neq 0 \\ \varphi_{t}\left(t_{0}, x_{0}\right) \leq M\left(D^{2} \varphi\left(t_{0}, x_{0}\right)\right)+c\left(x_{0}\right) u\left(t_{0}, x_{0}\right) & \text { if } D \varphi\left(t_{0}, x_{0}\right)=0 \\ \left(\text { resp. }, \varphi_{t}\left(t_{0}, x_{0}\right) \geq m\left(D^{2} \varphi\left(t_{0}, x_{0}\right)\right)+c\left(x_{0}\right) u\left(t_{0}, x_{0}\right)\right) . & \end{cases}
$$

(ii) For every $\left(t_{0}, x_{0}\right) \in(0,+\infty) \times \partial \Omega$, for all $\varphi \in C^{2}([0,+\infty) \times \bar{\Omega})$, such that $u-\varphi$ has a local maximum (resp., minimum) at $\left(t_{0}, x_{0}\right)$ and $D \varphi\left(t_{0}, x_{0}\right) \neq 0$, one has

$$
\left(\varphi_{t}\left(t_{0}, x_{0}\right)-\Delta_{\infty} \varphi\left(t_{0}, x_{0}\right)-c\left(x_{0}\right) u\left(t_{0}, x_{0}\right)\right) \wedge\left\langle D \varphi\left(t_{0}, x_{0}\right), \vec{n}\left(x_{0}\right)\right\rangle \leq 0
$$

(resp.,

$$
\left.\left(\varphi_{t}\left(t_{0}, x_{0}\right)-\Delta_{\infty} \varphi\left(t_{0}, x_{0}\right)-c\left(x_{0}\right) u\left(t_{0}, x_{0}\right)\right) \vee\left\langle D \varphi\left(t_{0}, x_{0}\right), \vec{n}\left(x_{0}\right)\right\rangle \geq 0\right) .
$$

Remark that if $\left(t_{0}, x_{0}\right) \in(0,+\infty) \times \partial \Omega$ and $D \varphi\left(t_{0}, x_{0}\right)=0$, then the boundary condition is satisfied.

We will show that if the principal eigenvalue of the stationary operator associated to (6.1) is positive, then $h$ decays to zero exponentially and that the rate of the decay depends on it. Let $\bar{\lambda}$ and $v$ be respectively the principal eigenvalue and a principal eigenfunction, i.e., $v$ is a positive solution of

$$
\begin{cases}\Delta_{\infty} v+(c(x)+\bar{\lambda}) v=0 & \text { in } \Omega \\ \frac{\partial v}{\partial \vec{n}}=0 & \text { on } \partial \Omega\end{cases}
$$


Proposition 6.2. Let $h \in C(\bar{\Omega} \times[0,+\infty))$ be a solution of (6.1) then

$$
\sup _{\Omega \times[0,+\infty)} \frac{h(t, x) \mathrm{e}^{\bar{\lambda} t}}{v(x)} \leq \sup _{\Omega} \frac{h_{0}^{+}(x)}{v(x)},
$$

where $h_{0}^{+}=\max \left\{h_{0}, 0\right\}$ denotes the positive part of $h_{0}$.

Proof. It suffices to prove that, fixed $\lambda<\bar{\lambda}$

$$
\sup _{[0, T) \times \Omega} \frac{h(t, x) \mathrm{e}^{\lambda t}}{v(x)} \leq \sup _{\Omega} \frac{h_{0}^{+}(x)}{v(x)}
$$

for any $T>0$. This implies that

$$
\sup _{[0, T) \times \Omega} \frac{h(t, x) \mathrm{e}^{\bar{\lambda} t}}{v(x)} \leq \sup _{\Omega} \frac{h_{0}^{+}(x)}{v(x)}
$$

for any $T>0$ and consequently (6.2). Let us denote $H(t, x)=h(t, x) \mathrm{e}^{\lambda t}$, it is easy to see that $H(t, x)$ satisfies

$$
\begin{cases}H_{t}=\Delta_{\infty} H+(c(x)+\lambda) H & \text { in }[0,+\infty) \times \Omega \\ \frac{\partial H}{\partial \vec{n}}=0 & \text { on }[0,+\infty) \times \partial \Omega \\ H(0, x)=h_{0}(x) & \text { for } x \in \Omega .\end{cases}
$$

Suppose by contradiction that there exists $T>0$ such that

$$
\gamma^{\prime}:=\sup _{[0, T) \times \Omega} \frac{h(t, x) \mathrm{e}^{\lambda t}}{v(x)}>\sup _{\Omega} \frac{h_{0}^{+}(x)}{v(x)}=: \bar{h} \geq 0 .
$$

Let us denote $w=\gamma v$, where

$$
\bar{h}<\gamma<\gamma^{\prime}
$$

and $\gamma$ is sufficiently close to $\gamma^{\prime}$ in order that

$$
\frac{\bar{\lambda} \frac{\gamma}{\gamma^{\prime}}-\lambda}{1-\frac{\gamma}{\gamma^{\prime}}}>|c|_{\infty}
$$

Since $\gamma<\gamma^{\prime}$, the function $H-w$ has a positive maximum on $[0, T] \times \bar{\Omega}$.

Fix $q>2, k>\frac{q}{2 r}$ and $\epsilon>0$ small, for $j \in \mathbb{N}$ we define the function

$$
\phi(t, x, s, y)=\left(\frac{j}{q}|x-y|^{q}+\frac{j}{2}|t-s|^{2}\right) \mathrm{e}^{-k(d(x)+d(y))}+\frac{\epsilon}{T-t},
$$

and we consider the supremum of

$$
H(t, x)-w(y)-\phi(t, x, s, y)
$$

over $([0, T) \times \bar{\Omega})^{2}$. Let $\left(t_{j}, x_{j}, s_{j}, y_{j}\right)$ be a point in $(\bar{\Omega} \times[0, T))^{2}$ where the maximum is attained. From

$$
H\left(t_{j}, x_{j}\right)-w\left(y_{j}\right)-\phi\left(t_{j}, x_{j}, t_{j}, y_{j}\right) \leq H\left(t_{j}, x_{j}\right)-w\left(y_{j}\right)-\phi\left(t_{j}, x_{j}, s_{j}, y_{j}\right)
$$

we deduce that

$$
t_{j}=s_{j}
$$


Let $(\widehat{t}, \widehat{x}) \in[0, T[\times \bar{\Omega}$ be such that $H(\widehat{t}, \widehat{x})-w(\widehat{x})=l>0$, then for $\epsilon$ small enough we have

$$
\frac{l}{2} \leq H(\widehat{t}, \widehat{x})-w(\widehat{x})-\frac{\epsilon}{T-\widehat{t}} \leq H\left(t_{j}, x_{j}\right)-w\left(y_{j}\right)-\frac{\epsilon}{T-t_{j}}-\frac{j}{q}\left|x_{j}-y_{j}\right|^{q} \mathrm{e}^{-k\left(d\left(x_{j}\right)+d\left(y_{j}\right)\right)} .
$$

Since $\frac{\epsilon}{T-t} \rightarrow+\infty$ as $t \uparrow T$, the previous inequality implies that, up to subsequence $\left(t_{j}, x_{j}, y_{j}\right) \rightarrow(\bar{t}, \bar{x}, \bar{x})$ as $j \rightarrow+\infty$ with $\bar{t}<T$ and that

Moreover

$$
H(\bar{t}, \bar{x})-w(\bar{x})>0
$$

and from (6.4) we deduce that

$$
\lim _{j \rightarrow+\infty} \frac{j}{q}\left|x_{j}-y_{j}\right|^{q}=0
$$

$$
w(\bar{x}) \geq \frac{\gamma}{\gamma^{\prime}} H(\bar{t}, \bar{x}) .
$$

Finally, since $\gamma>\bar{h}$, it is $\bar{t}>0$. Hence for $j$ large enough, $0<t_{j}<T$.

As in Theorem 4.8 the following holds true.

Claim 6.3. For $j$ large enough, we can choose $x_{j} \neq y_{j}$.

Indeed, suppose that $x_{j}=y_{j}$, then $\left(t_{j}, x_{j}\right)$ is a maximum point for

$$
U(t, x):=H(t, x)-\frac{\epsilon}{T-t}-\mathrm{e}^{-k d\left(x_{j}\right)}\left(\frac{j}{q}\left|x-x_{j}\right|^{q}+\frac{j}{2}\left|t-t_{j}\right|^{2}\right) \mathrm{e}^{-k d(x)},
$$

and a minimum point for

$$
W(t, x):=w(x)+\mathrm{e}^{-k d\left(x_{j}\right)}\left(\frac{j}{q}\left|x-x_{j}\right|^{q}+\frac{j}{2}\left|t-t_{j}\right|^{2}\right) \mathrm{e}^{-k d(x)} .
$$

We prove that $\left(t_{j}, x_{j}\right)$ is not both a strict local maximum and a strict local minimum. Indeed, in that case, if $H(t, x)-\frac{\epsilon}{T-t}$ is not locally constant around $\left(t_{j}, x_{j}\right)$, following the proof of Lemma 4.10, we can construct sequences $\left(t_{n}, x_{n}\right)_{n},\left(s_{n}, y_{n}\right)_{n}$ converging to $\left(t_{j}, x_{j}\right)$ as $n \rightarrow+\infty$, such that $\left(t_{n}, x_{n}\right) \neq\left(s_{n}, y_{n}\right)$ and

$$
\begin{aligned}
\varphi(t, x):= & C\left(\frac{\left|x-x_{n}\right|^{q}}{q}+\frac{\left|t-t_{n}\right|^{2}}{2}\right) \mathrm{e}^{-k d(x)}+\frac{\epsilon}{T-t}+H\left(s_{n}, y_{n}\right) \\
& -\frac{\epsilon}{T-s_{n}}-C\left(\frac{\left|y_{n}-x_{n}\right|^{q}}{q}+\frac{\left|s_{n}-t_{n}\right|^{2}}{2}\right) \mathrm{e}^{-k d\left(y_{n}\right)}
\end{aligned}
$$

is a test function for $H(t, x)$ at $\left(s_{n}, y_{n}\right)$, where $C=j \mathrm{e}^{-k d\left(x_{j}\right)}$. If $y_{n} \in \partial \Omega$, then

$$
\left\langle D \varphi\left(s_{n}, y_{n}\right), \vec{n}\left(y_{n}\right)\right\rangle \geq C\left[\left(\frac{k}{q}-\frac{1}{2 r}\right)\left|x_{n}-y_{n}\right|^{q}+\frac{k}{2}\left|s_{n}-t_{n}\right|^{2}\right]>0 .
$$

Then $D \varphi\left(s_{n}, y_{n}\right) \neq 0$ and by definition of subsolution

$$
\frac{\epsilon}{\left(T-s_{n}\right)^{2}}+C \mathrm{e}^{-k d\left(y_{n}\right)}\left(s_{n}-t_{n}\right) \leq \Delta_{\infty}\left(\varphi\left(s_{n}, y_{n}\right)\right)+\left(c\left(y_{n}\right)+\lambda\right) H\left(s_{n}, y_{n}\right) .
$$

If $y_{n}$ is an interior point and $D \varphi\left(s_{n}, y_{n}\right) \neq 0$, then again the previous inequality holds true, otherwise if $D \varphi\left(s_{n}, y_{n}\right)=0$, we have

$$
\frac{\epsilon}{\left(T-s_{n}\right)^{2}}+C \mathrm{e}^{-k d\left(y_{n}\right)}\left(s_{n}-t_{n}\right) \leq M\left(D^{2} \varphi\left(s_{n}, y_{n}\right)\right)+\left(c\left(y_{n}\right)+\lambda\right) H\left(s_{n}, y_{n}\right) .
$$


Passing to the limit as $n \rightarrow+\infty$, from both the previous relations we get

$$
\frac{\epsilon}{\left(T-t_{j}\right)^{2}} \leq\left(c\left(x_{j}\right)+\lambda\right) H\left(t_{j}, x_{j}\right) .
$$

By definition of subsolution, we get the same inequality if $H(t, x)-\frac{\epsilon}{T-t}$ is locally constant around $\left(t_{j}, x_{j}\right)$.

Proceeding in the same way, if either $w$ is locally constant around $x_{j}$ or not, since $\left(t_{j}, x_{j}\right)$ is a strict local minimum of $W(t, x)$, we get

$$
\left(c\left(x_{j}\right)+\bar{\lambda}\right) w\left(x_{j}\right) \leq 0 .
$$

Then, passing to the limit as $j \rightarrow+\infty$, we finally obtain

$$
(c(\bar{x})+\bar{\lambda}) w(\bar{x})<\frac{\epsilon}{(T-\bar{t})^{2}} \leq(c(\bar{x})+\lambda) H(\bar{t}, \bar{x}),
$$

which contradicts (6.5), (6.6) and (6.7).

Hence $\left(t_{j}, x_{j}\right)$ cannot be both a strict local maximum and a strict local minimum. In the first case, there exists $\left(s_{j}, y_{j}\right) \neq\left(t_{j}, x_{j}\right)$ such that

$$
\begin{aligned}
H\left(s_{j}, y_{j}\right)-w\left(x_{j}\right)-\frac{\epsilon}{T-s_{j}}-\left(\frac{j}{q}\left|x_{j}-y_{j}\right|^{q}+\frac{j}{2}\left|t_{j}-s_{j}\right|^{2}\right) \mathrm{e}^{-k\left(d\left(x_{j}\right)+d\left(y_{j}\right)\right)} & =H\left(t_{j}, x_{j}\right)-w\left(x_{j}\right)-\frac{\epsilon}{T-t_{j}} \\
= & \sup _{([0, T) \times \Omega)^{2}}(H(t, x)-w(y)-\phi(t, x, s, y)) .
\end{aligned}
$$

As before we get that $s_{j}=t_{j}$, then $x_{j} \neq y_{j}$ and this concludes the claim.

From the claim we deduce that $D_{x} \phi\left(t_{j}, x_{j}, t_{j}, y_{j}\right)$ and $D_{y} \phi\left(t_{j}, x_{j}, t_{j}, y_{j}\right)$ are different from 0 . Moreover there exist $X_{j}, Y_{j} \in S(N)$ satisfying (4.9) such that $\left(\frac{\epsilon}{\left(T-t_{j}\right)^{2}}, D_{x} \phi\left(t_{j}, x_{j}, t_{j}, y_{j}\right), X_{j}\right) \in \mathcal{P}^{2,+} H\left(t_{j}, x_{j}\right)$ and $\left(-D_{y} \phi\left(t_{j}, x_{j}, t_{j}, y_{j}\right), Y_{j}\right) \in J^{2,-} w\left(y_{j}\right)$. Now we can proceed as in the proof of Theorem 4.8 to obtain (6.8) and hence to reach a contradiction.

\section{REFERENCES}

[1] A. Anane, Simplicité et isolation de la première valeur propre du p-Laplacien avec poids. C. R. Acad. Sci. Paris Sér. I Math. 305 (1987) 752-728.

[2] G. Aronsson, M.G. Crandall and P. Juutinen, A tour of the theory of absolutely minimizing functions. Bull. Amer. Math. Soc. (N.S.) 41 (2004) 439-505.

[3] H. Berestycki, L. Nirenberg and S.R.S. Varadhan, The principal eigenvalue and maximum principle for second order elliptic operators in general domain. Comm. Pure Appl. Math. 47 (1994) 47-92.

[4] I. Birindelli and F. Demengel, Eigenvalue, maximum principle and regularity for fully nonlinear homogeneous operators. Comm. Pure Appl. Anal. 6 (2007) 335-366.

[5] J. Busca, M.J. Esteban, A. Quaas, Nonlinear eigenvalues and bifurcation problems for Pucci's operators. Ann. Inst. H. Poincaré Anal. Non Linéaire 22 (2005) 187-206.

[6] M.C. Crandall, H. Ishii and P.L. Lions, User's guide to viscosity solutions of second order partial differential equations. Bull. Amer. Math. Soc. (N.S.) 27 (1992) 1-67.

[7] L.C. Evans and W. Gangbo, Differential equations methods for the Monge-Kantorovich mass transfer problem, Mem. Amer. Math. Soc. 137. American Mathematical Society (1999).

[8] J. Garcia-Azorero, J.J. Manfredi, I. Peral and J.D. Rossi, Steklov eigenvalues for the $\infty$-Laplacian. Rend. Lincei Mat. Appl. 17 (2006) 199-210.

[9] H. Ishii and P.L. Lions, Viscosity solutions of fully nonlinear second-order elliptic partial differential equations. J. Diff. Equ. 83 (1990) 26-78.

[10] H. Ishii and Y. Yoshimura, Demi-eigenvalues for uniformly elliptic Isaacs operators. Preprint.

[11] P. Juutinen, Principal eigenvalue of a very badly degenerate operator and applications. J. Diff. Equ. 236 (2007) 532-550.

[12] P. Juutinen and B. Kawohl, On the evolution governed by the infinity Laplacian. Math. Ann. 335 (2006) 819-851.

[13] P. Juutinen, P. Lindqvist and J.J. Manfredi, The $\infty$-eigenvalue problem. Arch. Ration. Mech. Anal. 148 (1999) 89-105.

[14] P. Lindqvist, On a nonlinear eigenvalue problem. Report 68, Univ. Jyväskylä, Jyväskylä (1995) 33-54. 
[15] P.L. Lions, Bifurcation and optimal stochastic control. Nonlinear Anal. 7 (1983) 177-207.

[16] S. Patrizi, The Neumann problem for singular fully nonlinear operators. J. Math. Pures Appl. 90 (2008) $286-311$.

[17] S. Patrizi, Principal eigenvalues for Isaacs operators with Neumann boundary conditions. NoDEA 16 (2009) $79-107$.

[18] Y. Peres, O. Schramm, S. Sheffield and D. Wilson, Tug-of-war and the infinity Laplacian. J. Amer. Math. Soc. 22 (2009) $167-210$

[19] A. Quaas, Existence of positive solutions to a "semilinear" equation involving the Pucci's operators in a convex domain. Diff. Integral Equations 17 (2004) 481-494.

[20] A. Quaas and B. Sirakov, Principal eigenvalues and the Dirichlet problem for fully nonlinear operators. Adv. Math. 218 (2008) $105-135$. 